\title{
VASCULAR PLANT BIODIVERSITY OF FLOODPLAIN FOREST IN MORAVA AND DYJE RIVERS CONFLUENCE (FOREST DISTRICT SOUTOK), CZECH REPUBLIC
}

\author{
PETR MADĚRA*, RADOMÍR ŘRePKA, TOMÁŠ KOUTECKÝ, JAN ŠEBESTA \\ Faculty of Forestry and Wood Technology Mendel University in Brno, Zemédělská 3, 613 \\ 00, Brno, Czech Republic, *Corresponding author e-mail: petrmad@mendelu.cz.
}

Received: $21^{\text {th }}$ November 2018, Accepted: $10^{\text {th }}$ December 2018

\begin{abstract}
This paper presents an evaluation of full-area floristic investigation of floodplain forests in Soutok forest district (Židlochovice Forest State Enterprise) based on an individual forest stand inventory. The study area encompasses 5103 ha of forests, where 1186 segments were inventoried, and 71223 single records about presence of vascular plant species were done. We found 761 taxa (species, subspecies and hybrids), out of which 655 were herbs, 106 woody plants, 156 were endangered species and 177 adventive species. The average area of a segment was 4.3 ha. The mean number of species per segment was 64.42 in a range of 4- 180 .

Keywords: biodiversity, vascular plants, floodplain forest, forest district Soutok, Morava and Dyje rivers, Czech Republic
\end{abstract}

\section{INTRODUCTION}

The area under study is composed not only from valuable floodplain forests (Horák, 1961; Klimo et al. 2008; Maděra et al. 2011, 2013; Řepka et al. 2015) but there occur also the continental floodplain meadows (Vicherek et al., 2000) with solitary oak trees (Maděra et al., 2007) creating famous landscape character of the area. High abundance of many endangered xylophagous species of insect (Miklín et al., 2018, 2017; Miklín \& Č́ížek, 2014; Laštůvka et al., 2016), many rare bird species (Machar et al., 2018; Opluštil \& Čupa, 2012), amphibians (Šebela, 2004; Suchomel et al., 2017), invertebrates and other organisms (Hrib \& Kordiovský, 2004; Suchomel et al., 2017) due to the occurrence of well preserved habitats like large old trees, forest pools, riverine lakes, water channels and close nature floodplain forests, were reasons why a few small scale protected areas, NATURA 2000, UNESCO Biosphere Reserve were established in the area during last decades.

Floristic-oriented studies from the area of the confluence of the Morava and the Dyje rivers have been published only recently. Horák (1961) focused on the typology of floodplain forests, Vicherek et al. (2000) dealt with a floristic inventory in map squares regardless of forest or non-forest biotopes, Danihelka et al. (1995) and Danihelka \& Šumberová (2004) described the distribution of selected taxa in detail.

Presented paper is third part of articles concerning to vascular plant biodiversity evaluation in south Moravian floodplain forests. The previous were published by Maděra et al. (2011, 
2013) for forest districts Valtice and Tvrdonice. The aim of the work is to describe spatial distribution of vascular plants biodiversity in the area as a tool for both, responsible forest management and conservation efforts.

\section{MATERIAL AND METHODS}

\section{Study area}

The area of 5103 ha of floodplain forest in confluence of the Morava and Dyje rivers between towns Lanžhot and Břeclav was inventoried. This is the Soutok forest district, Židlochovice Forest Enterprise, which is a part of state forests managed by Lesy ČR s.p. The Morava and Dyje rivers in the studied area forms large alluvium and the border among the Czech Republic, Slovakia and Austria, respectively (Fig. 1).

\section{Fig. 1: Study area}

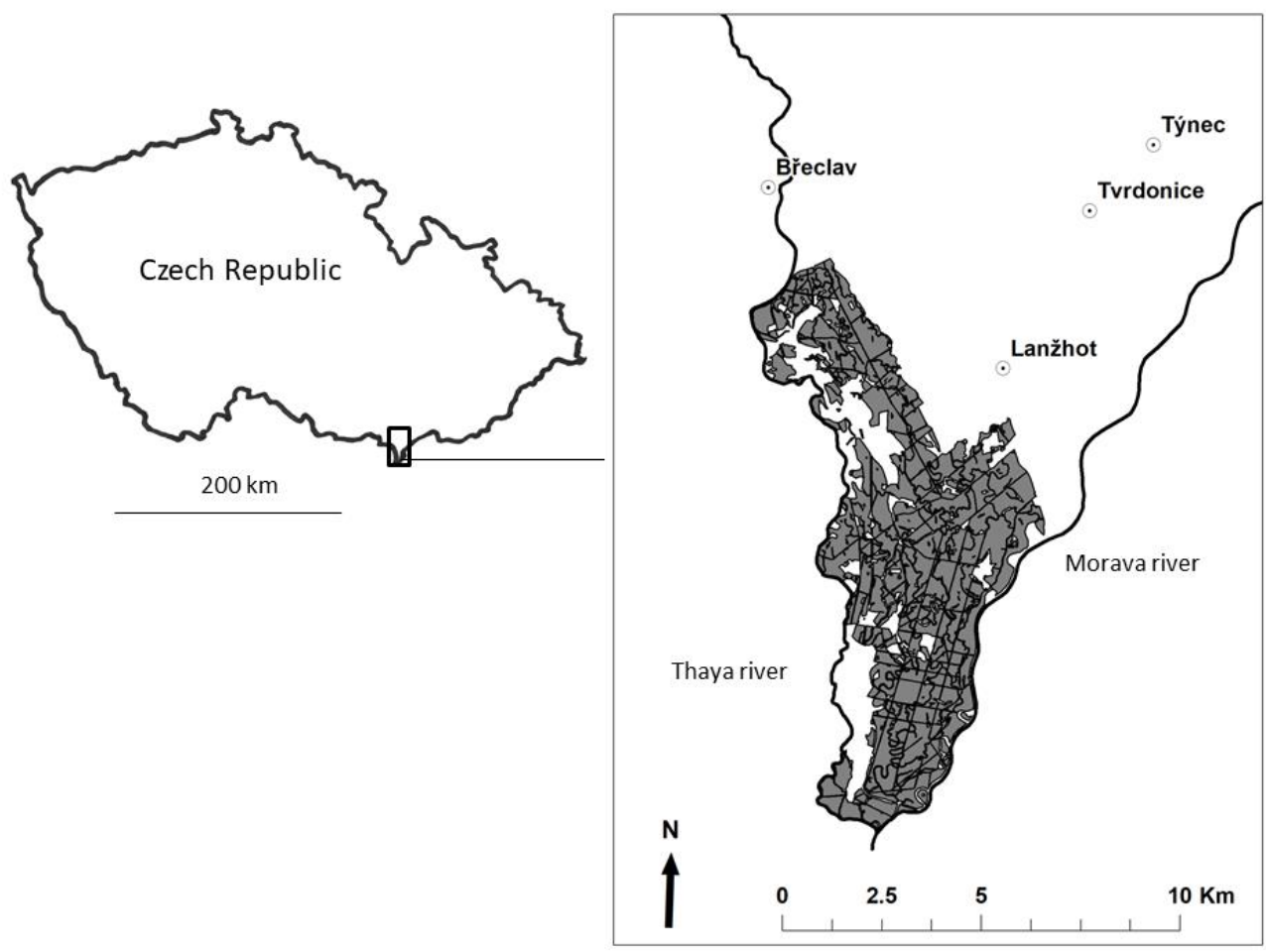

\section{METHODS}

All vascular plants in the area of the Soutok forest district were recorded between 2007 and 2011 down to the level of a segment; each segment corresponds to one stand group (exceptionally, similar groups are put together or non-homogeneous groups are divided). The presence of species in each segment is ticked in a list that includes 263 most common species of herbs in south-Moravian floodplains. Rare species and woody plants are added to the list. We followed nomenclature according to Kubát et al. (2002). The occurrence of species 
Maděra P. et al.: Vascular plant biodiversity of floodplain forest in Morava and Dyje rivers confluence (forest district Soutok), Czech Republic

growing only at the segment edges (stand adjacent to the forest roads, water channels, clearings and meadows) and dominant species (species of over $40 \%$ cover) are marked differently. The terrain survey needs to be conducted in two aspects: spring (March 20 May 31) and summer (June 1-November 30); also fresh clearings and young plantings were inventoried. The ticking lists are then transferred to a database and further processed. Both, list of alien plant species according to Pyšek et al. (2012a) and Red list according to Grulich $\&$ Chobot (2017) were used for evaluation of our dataset. The segments after digitalization become a site. The digitalization and creation of the species distribution maps was implemented in the GIS environment (ArcGIS).

\section{RESULTS}

The total study area was 5,103 ha of forest; 1,186 segments were explored and 71,223 records on the presence of vascular plant taxa were taken. According to the records, there are 761 species (and infraspecific taxa and hybrids) in the area, out of which there were 655 herbs and 106 woody plants. The mean size of a segment was 4.3 ha. On average, there were 64.42 taxa (range of 4-180) per segment (most segments containing 40-59 species). The numbers of species within a segment were distributed unequally - there were more segments with lower numbers of species than average (703) and fewer segments with higher numbers (483) (Fig. 2). On average, there were 8.94 species of woody plants and 55.48 species of herbs in a segment. The spatial distribution of the segments with their highlighted significance for biodiversity (the number of species per segment) is illustrated in Fig. 3.

\section{Fig. 2: Frequency of segments according to containing number of species}

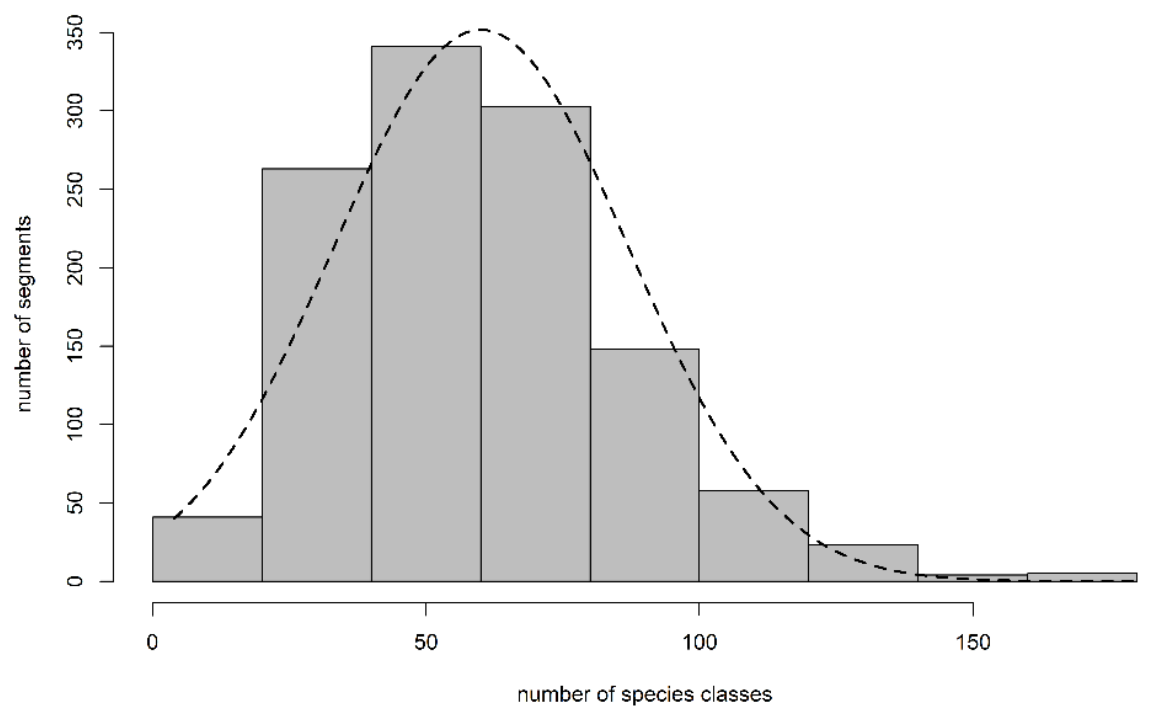


Fig. 3: Map of the number of all vascular plant species per segment in the study area
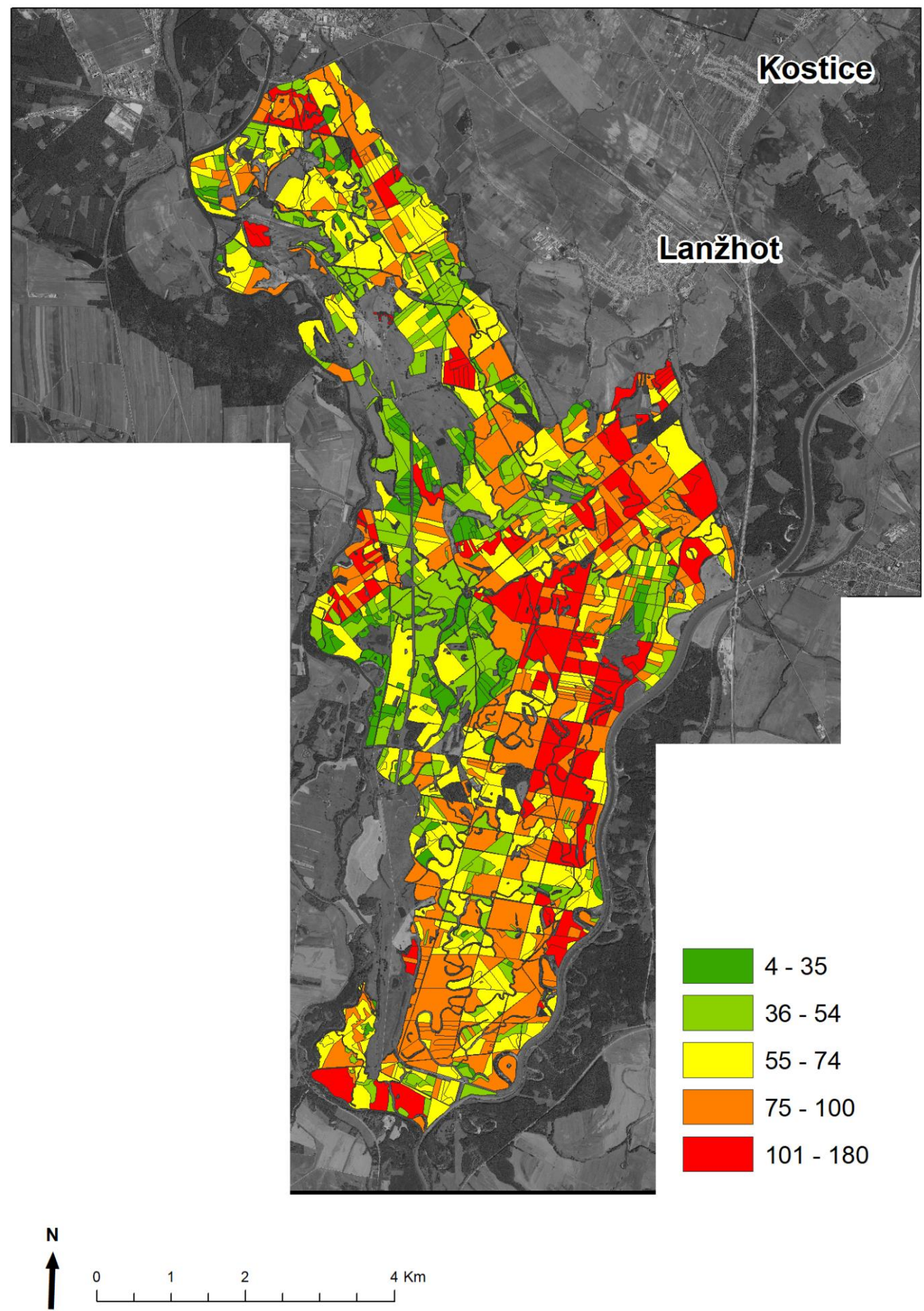
Maděra P. et al.: Vascular plant biodiversity of floodplain forest in Morava and Dyje rivers confluence (forest district Soutok), Czech Republic

We also examined the frequency of species occurrence (presence of a taxon in segments) in the study area (Table 1). The analysis shows that 126 species (i.e. nearly $17 \%$ ) occurred in one segment only, 343 species (i.e. $45 \%$ ) were present in 1-9 segments; it means that the species scarcely occurred in the area and were rare. The table also shows that another 251 species (i.e. $33 \%$ ) were present in 10-99 segments. These species can be referred to as scattered. 167 species (i.e. $22 \%$ ) were present in over 100 segments - these species were abundant. Only 20 species occurred in over $60 \%$ of segments (Table 2) - the species with high stability and diagnostic species of suballiance Ulmenion (Chytrý, 2013) only one adventive species is in this group - invasive neophyte Aster lanceolatus.

Table 1: The frequency of species occurrence in the study area

\begin{tabular}{|c|c|}
\hline Classes of segments number & Number of species \\
\hline $1000+$ & 4 \\
\hline $900-999$ & 3 \\
\hline $800-899$ & 9 \\
\hline $700-799$ & 4 \\
\hline $600-699$ & 8 \\
\hline $500-599$ & 21 \\
\hline $400-499$ & 11 \\
\hline $300-399$ & 15 \\
\hline $200-299$ & 31 \\
\hline $100-199$ & 61 \\
\hline $1-99$ & 597 \\
\hline $90-99$ & 14 \\
\hline $80-89$ & 7 \\
\hline $70-79$ & 17 \\
\hline $60-69$ & 16 \\
\hline $50-59$ & 17 \\
\hline $40-49$ & 18 \\
\hline $30-39$ & 26 \\
\hline $20-29$ & 42 \\
\hline $10-19$ & 94 \\
\hline $1-9$ & 343 \\
\hline
\end{tabular}


Table 2: The species with frequency over $60 \%$ of segments in the study area

\begin{tabular}{|l|c|}
\hline \multicolumn{1}{|c|}{ Species } & No. of segments \\
\hline Ficaria verna & 1080 \\
\hline Rubus caesius & 1065 \\
\hline Urtica dioica & 1046 \\
\hline Acer campestre & 1003 \\
\hline Quercus robur & 968 \\
\hline Fraxinus angustifolia & 911 \\
\hline Carex riparia & 908 \\
\hline Symphytum officinale & 890 \\
\hline Geum urbanum & 889 \\
\hline Glechoma hederacea & 868 \\
\hline Rumex sanguineus & 866 \\
\hline Aster lanceolatus & 854 \\
\hline Phalaris arundinacea & 839 \\
\hline Deschampsia cespitosa & 835 \\
\hline Brachypodium sylvaticum & 828 \\
\hline Lysimachia nummularia & 816 \\
\hline Galium aparine & 782 \\
\hline Ranunculus repens & 761 \\
\hline Iris pseudacorus & 755 \\
\hline Viola reichenbachiana & 733 \\
\hline
\end{tabular}

From the perspective of nature conservation, it is interesting to evaluate the proportion of adventive species (based on Pyšek et al., 2012a) and endangered species (based on Grulich \& Chobot, 2017). Considering merely the number of species (Fig. 4), almost a quarter (23.2\%, i.e. 177 taxa) were various categories of adventive species and $20.8 \%$ (156) taxa were species with various categories of conservation status. However, Fig.5 has a higher information capacity concerning the role of these groups in the study area. It shows the results categorised based on the number of records of the species in the segments. Based on this, the proportion of adventive species dropped to $14.8 \%$ (10,562 records) and the proportion of endangered species to $11.2 \%$ (7,948 records). 
Maděra P. et al.: Vascular plant biodiversity of floodplain forest in Morava and Dyje rivers confluence (forest district Soutok), Czech Republic

Fig. 4: Proportion of adventive, threatened and others vascular plant species in the study area

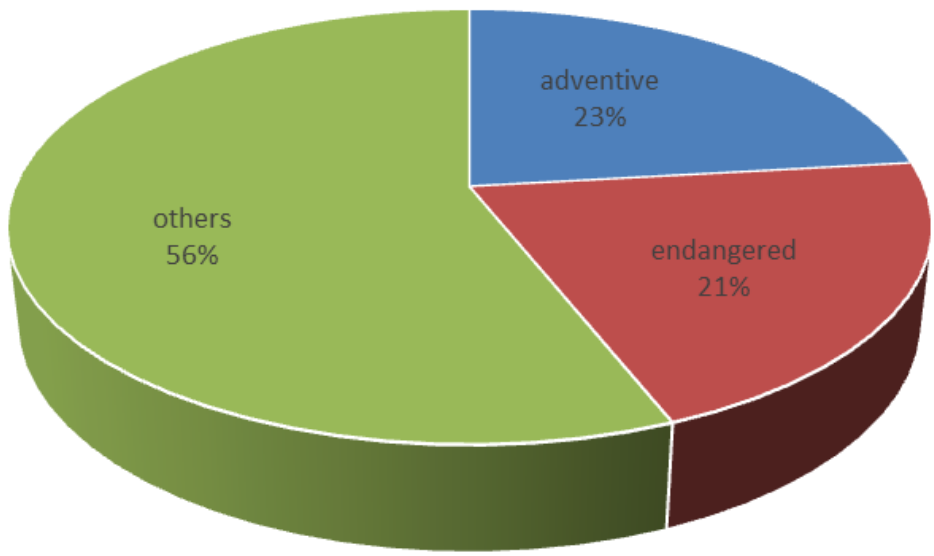

Fig. 5: Proportion of adventive, threatened and others vascular plant species in the study area according to the number of records

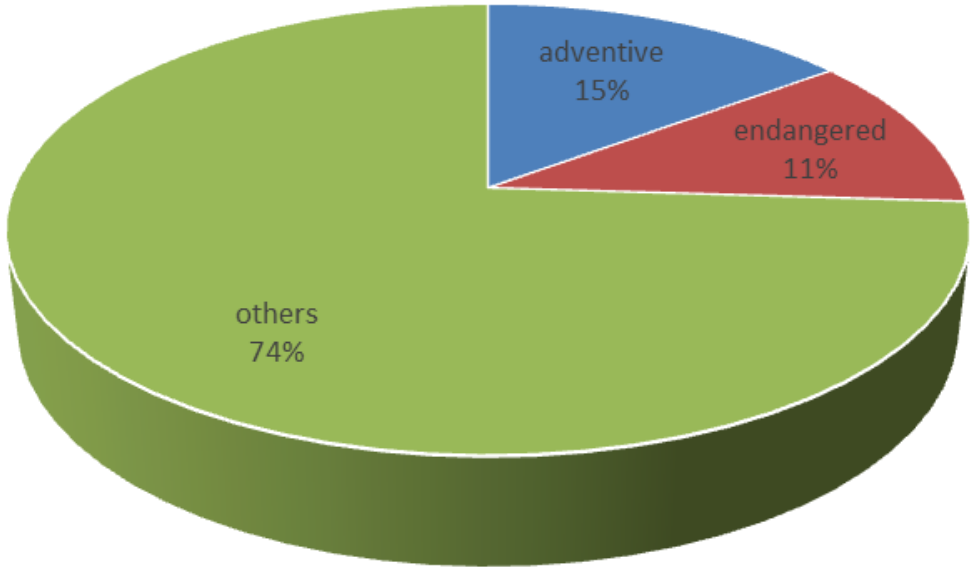

Within the set of adventive species, archeophytes $(54.8 \%)$ slightly prevailed over neophytes $(45.2 \%$ ); there were 38, i.e. $21.4 \%$ of invasive species in total (Fig. 6, Table 3). On average, there were 8 adventive species in a segment (range of 0-42). Only 27 segments contained no adventive species. There were up to $10 \%$ of adventive species in 440 segments, $10-20 \%$ in 579 segments, $20-30 \%$ in 126 segments, $31-40 \%$ in 14 segments, and no segment contains over $40 \%$ of adventive species. The loading of individual segments by the presence of adventive species is illustrated in the map (Fig. 7), neophytes especially are pictured in the map (Fig. 8). 
Table 3: The abundance of different categories of adventive species (according to Pyšek et al.,2012a). Arch $=$ archeophytes, neo $=$ neophytes, cas $=$ causal, nat $=$ naturalized, inv $=$ invasive

\begin{tabular}{|c|c|c|c|c|c|c|}
\hline \multirow[b]{2}{*}{$\begin{array}{c}\text { Adventive } \\
\text { species category }\end{array}$} & \multicolumn{2}{|c|}{ all species } & \multicolumn{2}{|c|}{ herbs } & \multicolumn{2}{|c|}{ woody plants } \\
\hline & $\begin{array}{l}\text { species } \\
\text { number }\end{array}$ & $\begin{array}{l}\text { records } \\
\text { number }\end{array}$ & $\begin{array}{l}\text { species } \\
\text { number }\end{array}$ & $\begin{array}{l}\text { records } \\
\text { number }\end{array}$ & $\begin{array}{l}\text { species } \\
\text { number }\end{array}$ & $\begin{array}{l}\text { records } \\
\text { number }\end{array}$ \\
\hline arch cas & 4 & 13 & 4 & 13 & 0 & 0 \\
\hline arch nat & 85 & 5235 & 79 & 4821 & 6 & 414 \\
\hline arch inv & 8 & 792 & 8 & 792 & 0 & 0 \\
\hline neo cas & 22 & 164 & 6 & 23 & 16 & 141 \\
\hline neo nat & 28 & 1017 & 24 & 931 & 4 & 86 \\
\hline neo inv & 30 & 3341 & 20 & 2922 & 10 & 419 \\
\hline
\end{tabular}

Fig. 6: Proportion of adventive species (classification according to Pyšek et al., 2012a) in the study area

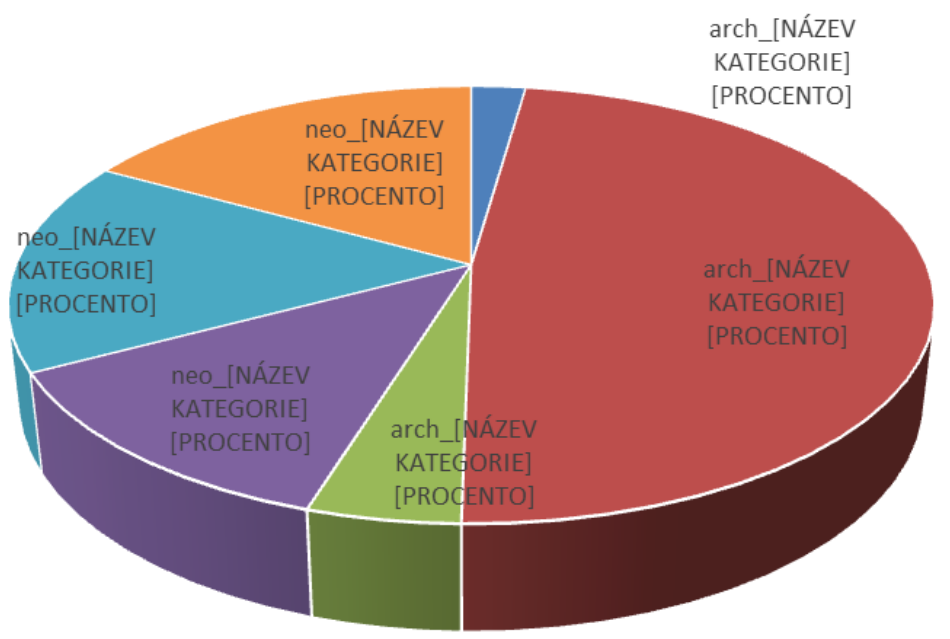


Maděra P. et al.: Vascular plant biodiversity of floodplain forest in Morava and Dyje rivers confluence (forest district Soutok), Czech Republic

Fig. 7: Map of the number of adventive species per segment in the study area

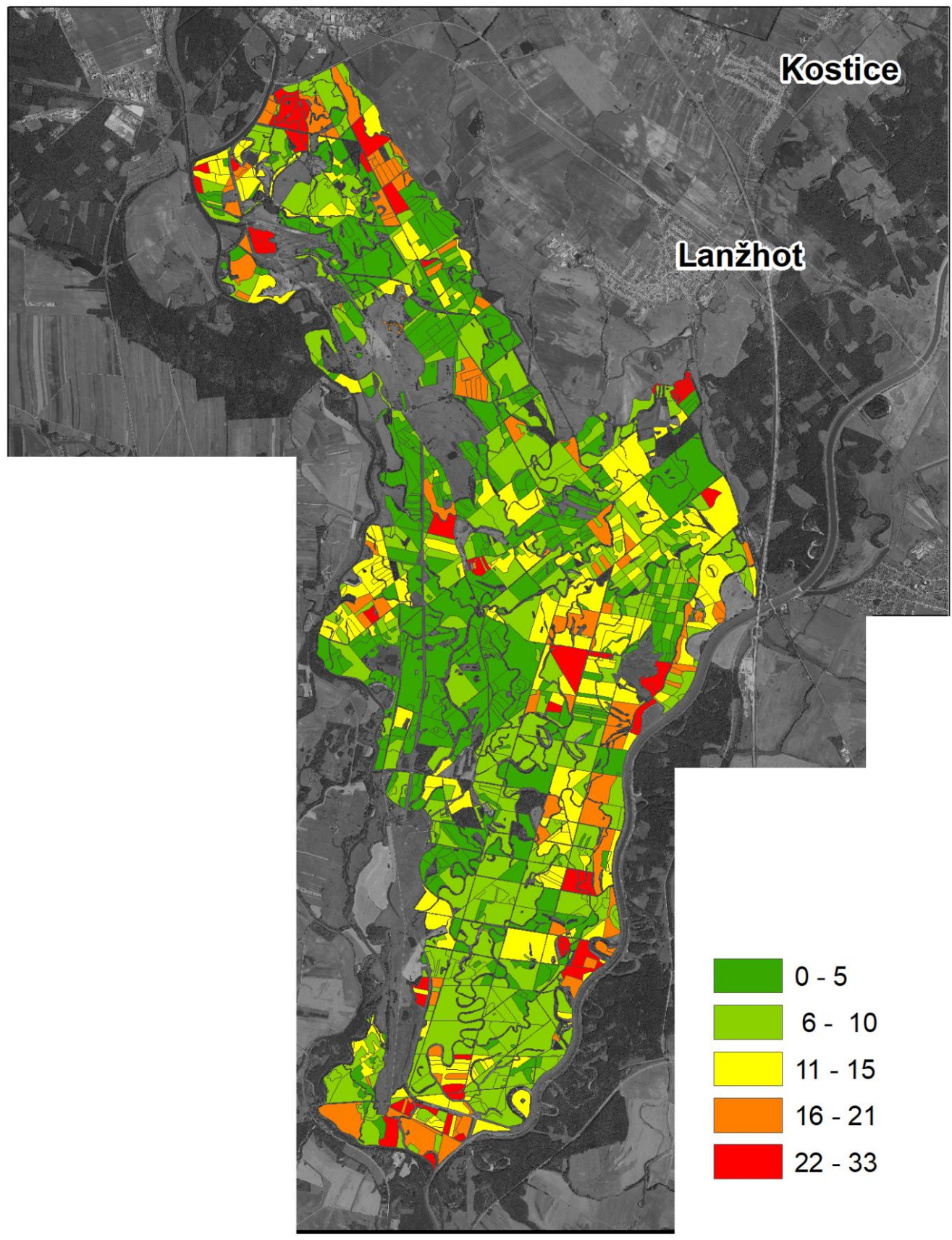

N

$4 \mathrm{Km}$ 
Fig. 8: Map of the number of neophytes per segment in the study area
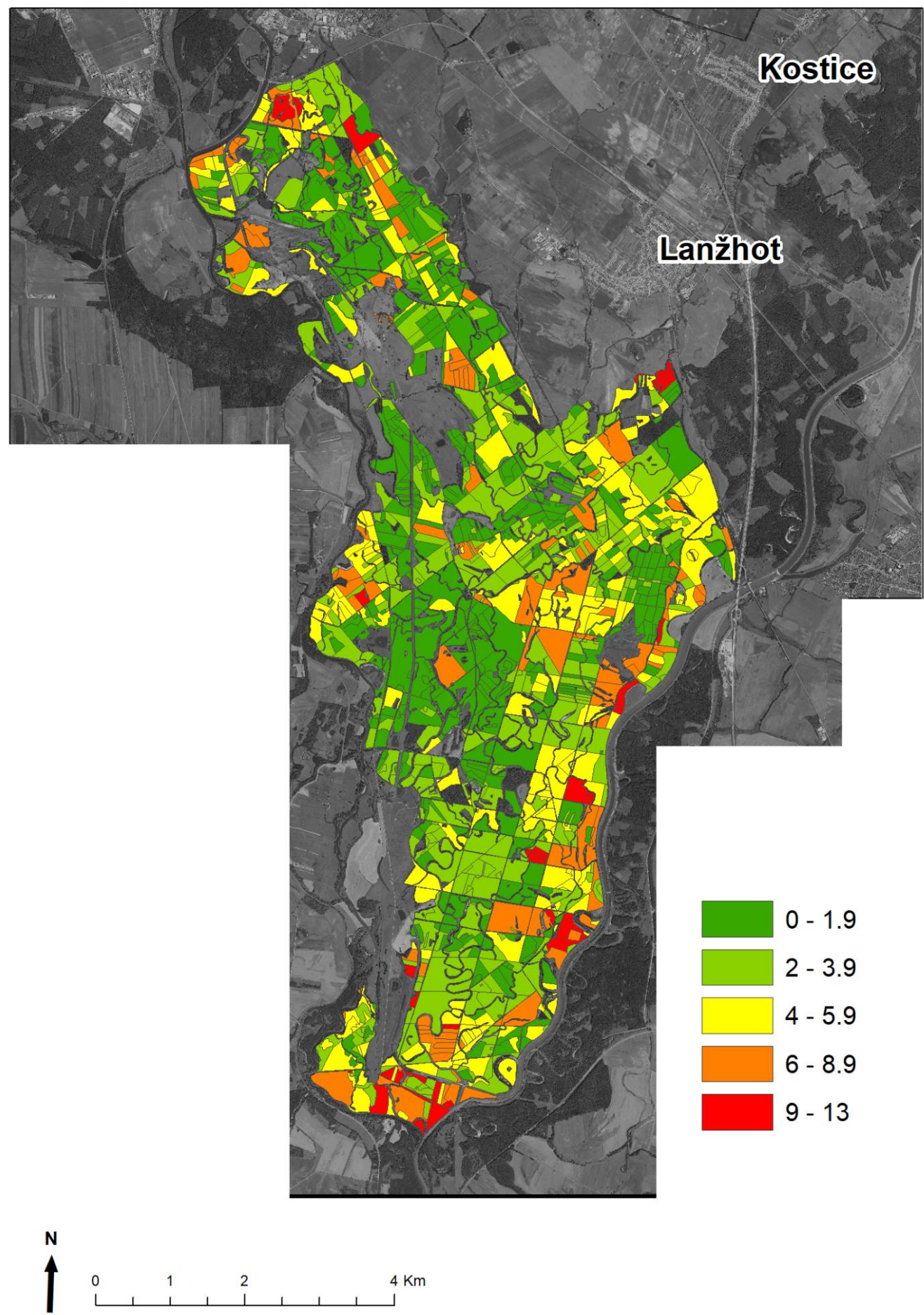
Maděra P. et al.: Vascular plant biodiversity of floodplain forest in Morava and Dyje rivers confluence (forest district Soutok), Czech Republic

As concerns endangered species, $20.75 \%$ of them were protected by law, the rest were within various categories of the Red List (Grulich \& Chobot, 2017). There were 18 critically endangered species, 34 strongly endangered and 44 endangered, the other 60 species were within C4 category - requiring further attention (Fig. 9, Table 4). The analysis shows that the mean number per segment was 5.4 of endangered species (range of 0-24). Endangered species were not present in 20 segments only; there were at least one endangered species in the other segments. Most segments (383) contain 6-9\% of endangered species; 76 segments even over $15 \%$. The most of endangered species $(62.2 \%)$ were present in 1-10 segments and only $11.3 \%$ of endangered species were present in over 100 segments. The spatial distribution of the numbers of endangered species of plants in the segments is shown in the map (Fig. 10). The map in Fig. 11 shows the species of categories $\mathrm{C} 1$ (critically endangered) and C2 (strongly endangered).

Table 4: The frequency of endangered species (according to Grulich \& Chobot, 2017) in the study area

\begin{tabular}{|c|c|c|c|c|c|c|}
\hline \multirow{2}{*}{$\begin{array}{c}\text { threat and protection } \\
\text { category }\end{array}$} & \multicolumn{2}{|c|}{ all species } & \multicolumn{2}{c|}{ herbs } & \multicolumn{2}{c|}{ woody plants } \\
\cline { 2 - 7 } & species & $\begin{array}{c}\text { records } \\
\text { number }\end{array}$ & $\begin{array}{c}\text { species } \\
\text { number }\end{array}$ & $\begin{array}{c}\text { records } \\
\text { number }\end{array}$ & $\begin{array}{c}\text { species } \\
\text { number }\end{array}$ & $\begin{array}{c}\text { records } \\
\text { number }\end{array}$ \\
\hline$\S 1$ & 11 & 237 & 11 & 237 & 0 & 0 \\
\hline$\S 2$ & 16 & 240 & 16 & 240 & 0 & 0 \\
\hline$\S 3$ & 4 & 30 & 3 & 28 & 1 & 2 \\
\hline $\mathrm{C} 1$ & 18 & 283 & 17 & 252 & 1 & 31 \\
\hline $\mathrm{C} 2$ & 34 & 1031 & 32 & 919 & 2 & 112 \\
\hline $\mathrm{C} 3$ & 44 & 1574 & 42 & 1505 & 2 & 69 \\
\hline $\mathrm{C} 4$ & 60 & 5060 & 51 & 2875 & 9 & 2185 \\
\hline
\end{tabular}

Fig. 9: Proportion of endangered species in the study area

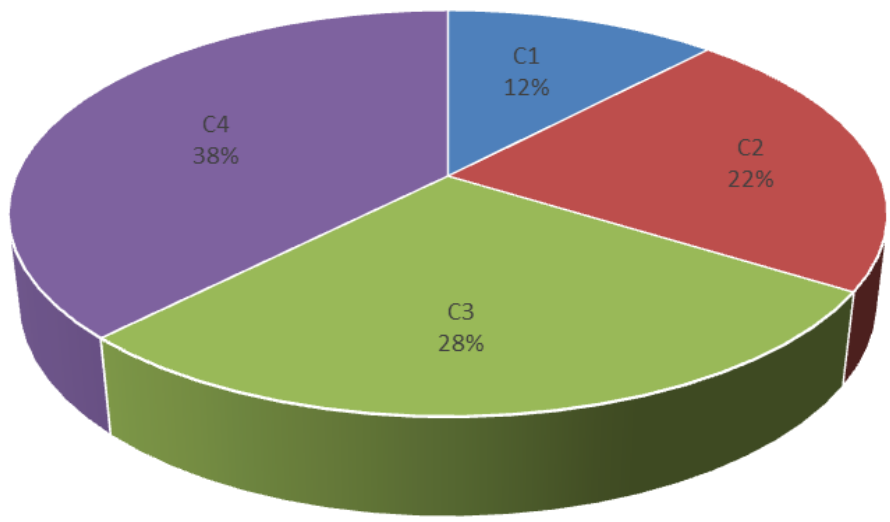


Fig. 10: Map of the number of endangered species per segment according to Grulich \& Chobot, (2017) in the study area
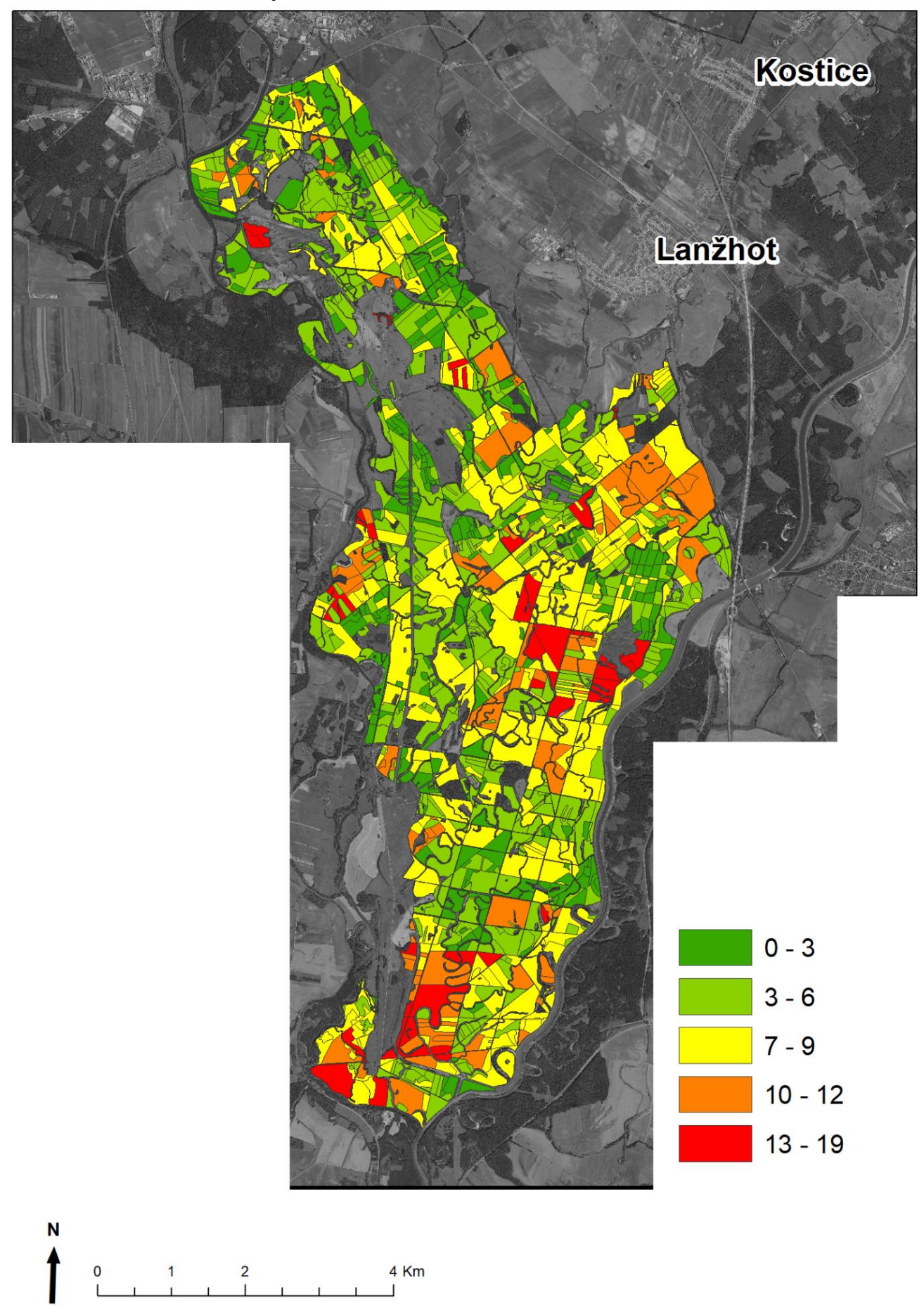
Maděra P. et al.: Vascular plant biodiversity of floodplain forest in Morava and Dyje rivers confluence (forest district Soutok), Czech Republic

Fig. 11: Map of the number of critical (C1) and strong endangered (C2) species per segment according to Grulich \& Chobot, (2017) in the study area.
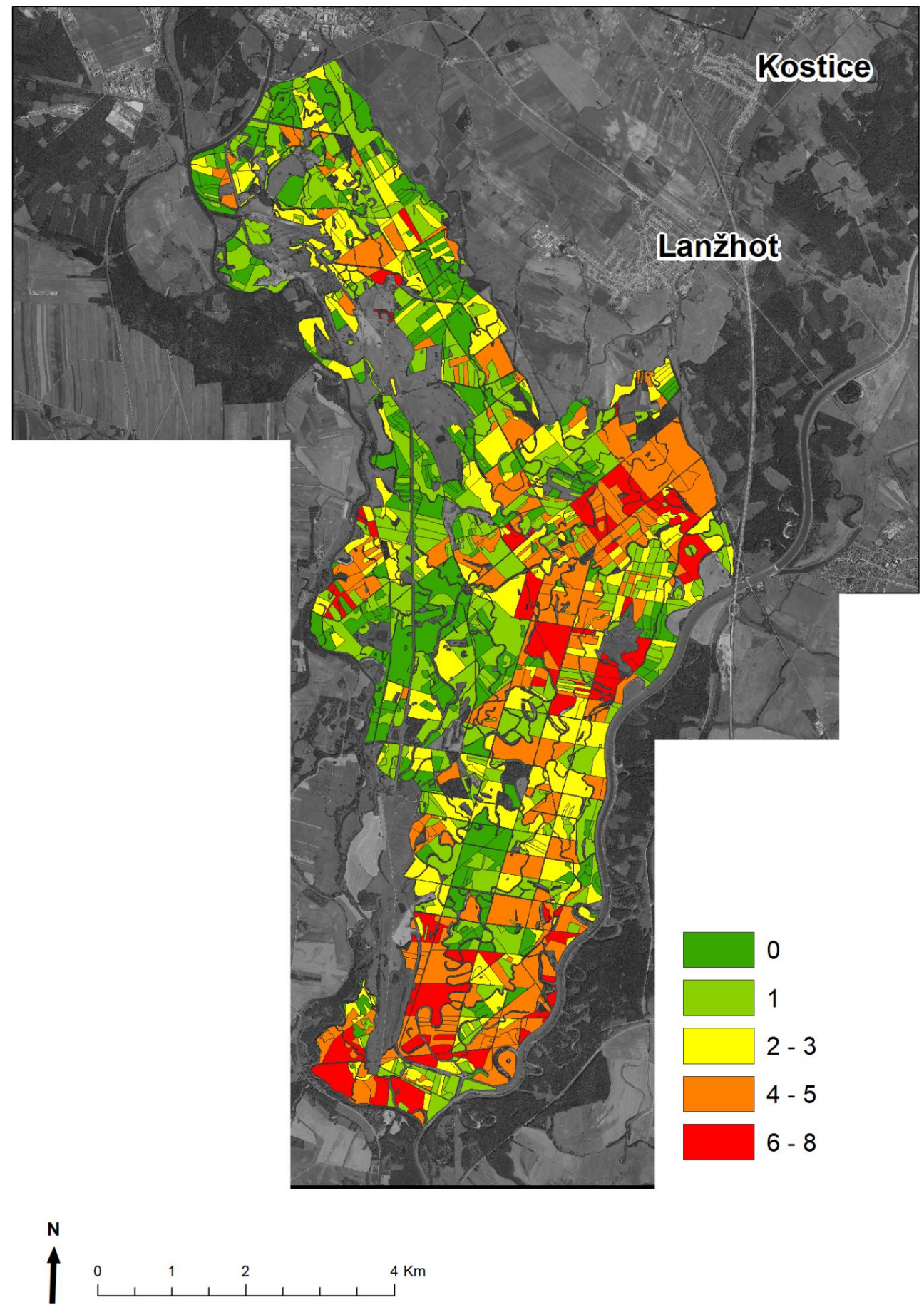


\section{Diversity of woody plants in the floodplain forests}

As has been mentioned above, we found 106 species, subspecies and hybrids of woody plants in the study area. Based on Úradníček et al. (2010), woody plants are not only trees and shrubs but also semi-shrubs (e.g. Vinca minor) or woody lianas (e.g. Vitis vinifera subsp. sylvestris) and shrublets, whose representative has not been found in the area (Fig.12).

Fig. 12: Proportion of occurrence of life forms of woody plants (according to Úradníček et al., 2010) in the study area.

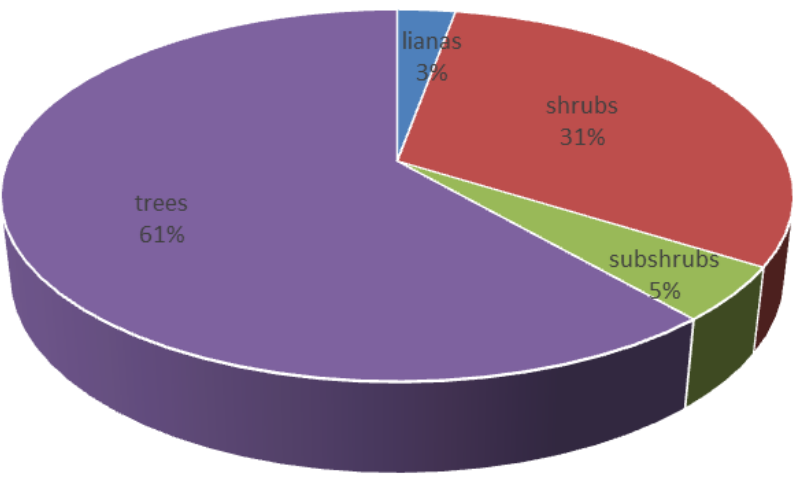

Out of the total number of woody plants found in the study area, there were 29 abundant species (occurrence in over 100 segments), 32 scattered species (10-99 segments) and 43 rare species (1-9 segments) - 18 species were recorded in one segment only.

From the perspective of autochthonous origin, 36 recorded species were various types of adventive species (Table 5). There are 10 recorded invasive neophytes, a more significant presence being recorded for both Acer negundo and Populus $\times$ canadensis - in nearly $13 \%$ of segments, the other species were spread less (under 3\% of segments). Pyšek et al. (2012a) also categorised the frequently grown Juglans nigra as an occasionally wild-growing neophyte; however, in the conditions of a floodplain forests, where is often planted, we can assume at least a very good naturalisation as it often regenerates naturally - it was recorded in $8.7 \%$ of segments.

Table 5: The presence of adventive woody plants species in segments (according to Pyšek et al., 2012a).

\begin{tabular}{|l|c|c|c|c|}
\hline \multicolumn{1}{|c|}{ Species } & $\begin{array}{c}\text { number of } \\
\text { segments }\end{array}$ & $\begin{array}{c}\text { proportion of } \\
\text { segments [\%] }\end{array}$ & \multicolumn{2}{|c|}{ adventive species category } \\
\hline Pyrus communis & 268 & 22,6 & arch & naturalized \\
\hline Malus domestica & 88 & 7,4 & arch & naturalized \\
\hline Malus $\times$ dasyphylla & 48 & 4,0 & arch & naturalized \\
\hline Juglans regia & 4 & 0,3 & arch & naturalized \\
\hline Prunus insititia & 4 & 0,3 & arch & naturalized \\
\hline Prunus domestica & 2 & 0,2 & arch & naturalized \\
\hline Juglans nigra & 103 & 8,7 & neo & casual \\
\hline
\end{tabular}


Maděra P. et al.: Vascular plant biodiversity of floodplain forest in Morava and Dyje rivers confluence (forest district Soutok), Czech Republic

\begin{tabular}{|c|c|c|c|c|}
\hline Morus alba & 11 & 0,9 & neo & casual \\
\hline Fraxinus ornus & 7 & 0,6 & neo & casual \\
\hline Picea pungens & 4 & 0,3 & neo & casual \\
\hline Castanea sativa & 2 & 0,2 & neo & casual \\
\hline Catalpa bignonioides & 2 & 0,2 & neo & casual \\
\hline Gleditsia triacanthos & 2 & 0,2 & neo & casual \\
\hline Tilia tomentosa & 2 & 0,2 & neo & casual \\
\hline Acer saccharinum & 1 & 0,1 & neo & casual \\
\hline Hibicus syriacus & 1 & 0,1 & neo & casual \\
\hline Phellodendron amurense & 1 & 0,1 & neo & casual \\
\hline Platanus $\times$ hispanica & 1 & 0,1 & neo & casual \\
\hline Populus candicans & 1 & 0,1 & neo & casual \\
\hline Rosa multiflora & 1 & 0,1 & neo & casual \\
\hline Thuja plicata & 1 & 0,1 & neo & casual \\
\hline Zelkova serrata & 1 & 0,1 & neo & casual \\
\hline Aesculus hippocastanum & 58 & 4,9 & neo & naturalized \\
\hline Pinus nigra & 16 & 1,3 & neo & naturalized \\
\hline Ribes rubrum & 11 & 0,9 & neo & naturalized \\
\hline Pseudotsuga menziesii & 1 & 0,1 & neo & naturalized \\
\hline Populus $\times$ canadensis & 153 & 12,9 & neo & invasive \\
\hline Acer negundo & 152 & 12,8 & neo & invasive \\
\hline Robinia pseudacacia & 34 & 2,9 & neo & invasive \\
\hline Parthenocissus inserta & 27 & 2,3 & neo & invasive \\
\hline Quercus rubra & 19 & 1,6 & neo & invasive \\
\hline Prunus cerasifera & 16 & 1,3 & neo & invasive \\
\hline Fraxinus pennsylvanica & 12 & 1,0 & neo & invasive \\
\hline Ailanthus altissima & 4 & 0,3 & neo & invasive \\
\hline Amorpha fruticosa & 1 & 0,1 & neo & invasive \\
\hline Prunus serotina & 1 & 0,1 & neo & invasive \\
\hline
\end{tabular}

14 species of the woody plants fall within threatened species of some category (Table 6) but only Cornus mas is protected by law and it was found in two segments only. Floodplain forests are indispensable biotopes of critically endangered woody species Populus nigra (31 segments), endangered species Malus sylvestris (68 segments), vulnerable species Fraxinus angustifolia (911 segments) and Pyrus pyraster (248). There is also a strong population of elms, both Ulmus laevis and U. minor (517 and 391 segments, respectively). High presence of Quercus cerris is due to its artificial plantation in dryer sandy sites. 
Table 6: The presence of threatened woody plant species (according to Grulich \& Chobot, 2017).

\begin{tabular}{|l|c|c|}
\hline \multicolumn{1}{|c|}{ species } & $\begin{array}{c}\text { number of } \\
\text { segments }\end{array}$ & $\begin{array}{c}\text { threat } \\
\text { category }\end{array}$ \\
\hline Populus nigra & 31 & $\mathrm{C} 1 \mathrm{t}$ \\
\hline Sorbus aria & 2 & $\mathrm{C} 2 \mathrm{~b}$ \\
\hline Quercus cerris & 110 & $\mathrm{C} 2 \mathrm{r}$ \\
\hline Malus sylvestris & 68 & $\mathrm{C} 3$ \\
\hline Rosa tomentosa & 1 & $\mathrm{C} 3$ \\
\hline Cornus mas & 2 & $\mathrm{C} 4 \mathrm{a}, \S 3$ \\
\hline Fraxinus angustifolia & 911 & $\mathrm{C} 4 \mathrm{a}$ \\
\hline Loranthus europaeus & 108 & $\mathrm{C} 4 \mathrm{a}$ \\
\hline Pyrus pyraster & 248 & $\mathrm{C} 4 \mathrm{a}$ \\
\hline Thymus pannonicus & 3 & $\mathrm{C} 4 \mathrm{a}$ \\
\hline Ulmus laevis & 517 & $\mathrm{C} 4 \mathrm{a}$ \\
\hline Ulmus minor & 391 & $\mathrm{C} 4 \mathrm{a}$ \\
\hline Viscum album subsp. austriacum & 4 & $\mathrm{C} 4 \mathrm{a}$ \\
\hline Quercus polycarpa & 1 & $\mathrm{C} 4 \mathrm{~b}$ \\
\hline
\end{tabular}

\section{Diversity of herbs in the floodplain forests}

We determined 655 species, subspecies and hybrids of herbs in the floodplain forest herb layer. Out of the total number of herbs found, there were 138 abundant species (occurrence in over 100 segments), 218 scattered species (10-99 segments) and 299 rare species within the study area (1-9 segments) - 107 species were found in one segment only.

From the perspective of autochthonous origin, 141 recorded species were various types of adventive species (Table 7), out of which there were 91 archeophytes and 50 neophytes, 28 invasive species. The more significant invasive archeophytes was Cirsium arvense in $56.5 \%$ of segments which was dominant in forest edges and clearings. The most significant and highly aggressive invasive neophytes in the area was Aster lanceolatus, whose presence $72 \%$ of segments and frequent dominance in younger and older stands of the floodplain forest presents a problem with almost no solution any more (̌̌epka \& Maděra, 2009a). The other abundant invasive neophytes in the area were Bidens frondosa (50\% of segments), Impatiens parviflora (29\%), Conyza canadensis (26\%), Erigeron annuus (19.6\%) and Echinocystis lobata (16.2\%) usually grew in clearings and newly established cultures and only the first two mentioned ones penetrated into forest communities. 
Maděra P. et al.: Vascular plant biodiversity of floodplain forest in Morava and Dyje rivers confluence (forest district Soutok), Czech Republic

Table 7: The presence of adventive herb species in segments (according to Pyšek et al., 2012a).

\begin{tabular}{|c|c|c|c|c|}
\hline Species & $\begin{array}{c}\text { number of } \\
\text { segments }\end{array}$ & $\begin{array}{l}\text { proportion of } \\
\text { segments [\%] }\end{array}$ & \multicolumn{2}{|c|}{ adventive species category } \\
\hline Xanthium strumarium & 7 & 0,6 & $\operatorname{arc}$ & casual \\
\hline Panicum miliaceum & 3 & 0,3 & $\operatorname{arc}$ & casual \\
\hline Triticum aestivum & 2 & 0,2 & $\operatorname{arc}$ & casual \\
\hline Beta vulgaris & 1 & 0,1 & $\operatorname{arc}$ & casual \\
\hline Descurainia sophia & 835 & 70,4 & $\operatorname{arc}$ & naturalized \\
\hline Lapsana communis & 568 & 47,9 & $\operatorname{arc}$ & naturalized \\
\hline Arctium lappa & 558 & 47,0 & $\operatorname{arc}$ & naturalized \\
\hline Veronica hederifolia & 426 & 35,9 & $\operatorname{arc}$ & naturalized \\
\hline Tanacetum vulgare & 266 & 22,4 & $\operatorname{arc}$ & naturalized \\
\hline Tripleurospermum inodorum & 205 & 17,3 & $\operatorname{arc}$ & naturalized \\
\hline Setaria pumila & 175 & 14,8 & $\operatorname{arc}$ & naturalized \\
\hline Sonchus asper & 126 & 10,6 & $\operatorname{arc}$ & naturalized \\
\hline Capsella bursa-pastoris & 124 & 10,5 & $\operatorname{arc}$ & naturalized \\
\hline Atriplex patula & 123 & 10,4 & $\operatorname{arc}$ & naturalized \\
\hline Lactuca serriola & 115 & 9,7 & $\operatorname{arc}$ & naturalized \\
\hline Lamium purpureum & 91 & 7,7 & $\operatorname{arc}$ & naturalized \\
\hline Ballota nigra & 72 & 6,1 & $\operatorname{arc}$ & naturalized \\
\hline Anchusa officinalis & 70 & 5,9 & $\operatorname{arc}$ & naturalized \\
\hline Sonchus arvensis & 66 & 5,6 & $\operatorname{arc}$ & naturalized \\
\hline Silene latifolia subsp. alba & 65 & 5,5 & $\operatorname{arc}$ & naturalized \\
\hline Linaria vulgaris & 62 & 5,2 & arc & naturalized \\
\hline Chelidonium majus & 58 & 4,9 & $\operatorname{arc}$ & naturalized \\
\hline Solanum nigrum & 58 & 4,9 & $\operatorname{arc}$ & naturalized \\
\hline Carduus acanthoides & 56 & 4,7 & $\operatorname{arc}$ & naturalized \\
\hline Fallopia convolvulus & 56 & 4,7 & $\operatorname{arc}$ & naturalized \\
\hline Geranium pusillum & 51 & 4,3 & $\operatorname{arc}$ & naturalized \\
\hline Convolvulus arvensis & 46 & 3,9 & $\operatorname{arc}$ & naturalized \\
\hline Setaria viridis & 46 & 3,9 & $\operatorname{arc}$ & naturalized \\
\hline Bromus sterilis & 40 & 3,4 & $\operatorname{arc}$ & naturalized \\
\hline Bromus hordeaceus & 38 & 3,2 & arc & naturalized \\
\hline Viola odorata & 35 & 3,0 & $\operatorname{arc}$ & naturalized \\
\hline Lamium album & 32 & 2,7 & $\operatorname{arc}$ & naturalized \\
\hline Sonchus oleraceus & 30 & 2,5 & $\operatorname{arc}$ & naturalized \\
\hline
\end{tabular}


Journal of Landscape Ecology (2018), Vol: 11 / No. 3

\begin{tabular}{|c|c|c|c|c|}
\hline Senecio vulgaris & 28 & 2,4 & $\operatorname{arc}$ & naturalized \\
\hline Veronica arvensis & 27 & 2,3 & $\operatorname{arc}$ & naturalized \\
\hline Digitaria sanguinalis & 20 & 1,7 & arc & naturalized \\
\hline Saponaria officinalis & 17 & 1,4 & $\operatorname{arc}$ & naturalized \\
\hline Bromus japonicus & 16 & 1,3 & arc & naturalized \\
\hline Berteroa incana & 15 & 1,3 & $\operatorname{arc}$ & naturalized \\
\hline Arctium tomentosum & 14 & 1,2 & $\operatorname{arc}$ & naturalized \\
\hline Myosotis arvensis & 13 & 1,1 & arc & naturalized \\
\hline Vicia villosa & 13 & 1,1 & arc & naturalized \\
\hline Vicia angustifolia & 12 & 1,0 & $\operatorname{arc}$ & naturalized \\
\hline Melilotus albus & 11 & 0,9 & arc & naturalized \\
\hline Verbena officinalis & 11 & 0,9 & $\operatorname{arc}$ & naturalized \\
\hline Lathyrus tuberosus & 9 & 0,8 & arc & naturalized \\
\hline Tragopogon dubius & 9 & 0,8 & arc & naturalized \\
\hline Bromus commutatus & 8 & 0,7 & $\operatorname{arc}$ & naturalized \\
\hline Melilotus officinalis & 8 & 0,7 & $\operatorname{arc}$ & naturalized \\
\hline Sisymbrium officinale & 8 & 0,7 & arc & naturalized \\
\hline Thlaspi arvense & 7 & 0,6 & $\operatorname{arc}$ & naturalized \\
\hline Leonurus cardiaca s.lat. & 6 & 0,5 & $\operatorname{arc}$ & naturalized \\
\hline Bromus tectorum & 5 & 0,4 & arc & naturalized \\
\hline Viola tricolor & 5 & 0,4 & $\operatorname{arc}$ & naturalized \\
\hline Anagallis arvensis & 4 & 0,3 & arc & naturalized \\
\hline Artemisia absinthium & 4 & 0,3 & arc & naturalized \\
\hline Chenopodium botrys & 4 & 0,3 & arc & naturalized \\
\hline Erodium cicutarium & 4 & 0,3 & arc & naturalized \\
\hline Papaver rhoeas & 4 & 0,3 & $\operatorname{arc}$ & naturalized \\
\hline Vicia sativa & 4 & 0,3 & arc & naturalized \\
\hline Armoracia rusticana & 3 & 0,3 & $\operatorname{arc}$ & naturalized \\
\hline Avena sativa & 3 & 0,3 & arc & naturalized \\
\hline Cynodon dactylon & 3 & 0,3 & arc & naturalized \\
\hline Lamium amplexicaule & 3 & 0,3 & arc & naturalized \\
\hline Microrrhinum minus & 3 & 0,3 & $\operatorname{arc}$ & naturalized \\
\hline Sambucus ebulus & 3 & 0,3 & $\operatorname{arc}$ & naturalized \\
\hline Vicia villosa subsp. varia & 3 & 0,3 & arc & naturalized \\
\hline Crepis capillaris & 2 & 0,2 & $\operatorname{arc}$ & naturalized \\
\hline Erysimum cheiranthoides & 2 & 0,2 & arc & naturalized \\
\hline
\end{tabular}


Maděra P. et al.: Vascular plant biodiversity of floodplain forest in Morava and Dyje rivers confluence (forest district Soutok), Czech Republic

\begin{tabular}{|c|c|c|c|c|}
\hline Geranium columbinum & 2 & 0,2 & arc & naturalized \\
\hline Lithospermum arvense & 2 & 0,2 & $\operatorname{arc}$ & naturalized \\
\hline Mentha $\times$ verticillata & 2 & 0,2 & $\operatorname{arc}$ & naturalized \\
\hline Atriplex tatarica & 1 & 0,1 & $\operatorname{arc}$ & naturalized \\
\hline Cichorium intybus & 1 & 0,1 & arc & naturalized \\
\hline Crepis setosa & 1 & 0,1 & arc & naturalized \\
\hline Euphorbia peplus & 1 & 0,1 & $\operatorname{arc}$ & naturalized \\
\hline Geranium dissectum & 1 & 0,1 & arc & naturalized \\
\hline Hyoscyamus niger & 1 & 0,1 & $\operatorname{arc}$ & naturalized \\
\hline Lepidium ruderale & 1 & 0,1 & arc & naturalized \\
\hline Malva neglecta & 1 & 0,1 & $\operatorname{arc}$ & naturalized \\
\hline Onopordum acanthium & 1 & 0,1 & $\operatorname{arc}$ & naturalized \\
\hline Parietaria officinalis & 1 & 0,1 & $\operatorname{arc}$ & naturalized \\
\hline Veronica polita & 1 & 0,1 & $\operatorname{arc}$ & naturalized \\
\hline Cirsium arvense & 670 & 56,5 & $\operatorname{arc}$ & invasive \\
\hline Portulaca oleracea & 42 & 3,5 & $\operatorname{arc}$ & invasive \\
\hline Echinochloa crus-galli & 29 & 2,4 & arc & invasive \\
\hline Eragrostis minor & 24 & 2,0 & arc & invasive \\
\hline Atriplex sagittata & 11 & 0,9 & $\operatorname{arc}$ & invasive \\
\hline Chenopodium pedunculare & 8 & 0,7 & arc & invasive \\
\hline Digitaria ischaemum & 7 & 0,6 & $\operatorname{arc}$ & invasive \\
\hline Conium maculatum & 1 & 0,1 & $\operatorname{arc}$ & invasive \\
\hline Xanthium italicum & 16 & 1,3 & neo & casual \\
\hline Hemerocallis fulva & 2 & 0,2 & neo & casual \\
\hline Lycopersicum esculentum & 2 & 0,2 & neo & casual \\
\hline Helianthus annuus & 1 & 0,1 & neo & casual \\
\hline Phacelia tanacetifolia & 1 & 0,1 & neo & casual \\
\hline Phytolacca americana & 1 & 0,1 & neo & casual \\
\hline Oxalis fontana & 364 & 30,7 & neo & naturalized \\
\hline Galega officinalis & 178 & 15,0 & neo & naturalized \\
\hline Trifolium hybridum & 125 & 10,5 & neo & naturalized \\
\hline Juncus tenuis & 48 & 4,0 & neo & naturalized \\
\hline Chenopodium strictum & 46 & 3,9 & neo & naturalized \\
\hline Epilobium ciliatum & 41 & 3,5 & neo & naturalized \\
\hline Datura stramonium & 24 & 2,0 & neo & naturalized \\
\hline Amaranthus albus & 15 & 1,3 & neo & naturalized \\
\hline
\end{tabular}


Journal of Landscape Ecology (2018), Vol: 11 / No. 3

\begin{tabular}{|c|c|c|c|c|}
\hline Sagittaria latifolia & 15 & 1,3 & neo & naturalized \\
\hline Rumex thyrsiflorus & 13 & 1,1 & neo & naturalized \\
\hline Chenopodium pumilio & 12 & 1,0 & neo & naturalized \\
\hline Erechtites hieraciifolia & 12 & 1,0 & neo & naturalized \\
\hline Asclepias syriaca & 10 & 0,8 & neo & naturalized \\
\hline Agrostis gigantea & 7 & 0,6 & neo & naturalized \\
\hline Senecio vernalis & 6 & 0,5 & neo & naturalized \\
\hline Oenothera biennis & 4 & 0,3 & neo & naturalized \\
\hline Geranium pyrenaicum & 2 & 0,2 & neo & naturalized \\
\hline Rubus armeniacus & 2 & 0,2 & neo & naturalized \\
\hline Veronica persica & 2 & 0,2 & neo & naturalized \\
\hline Alcea rosea & 1 & 0,1 & neo & naturalized \\
\hline Medicago sativa & 1 & 0,1 & neo & naturalized \\
\hline Ornithogalum nutans & 1 & 0,1 & neo & naturalized \\
\hline Pleioblastus chino & 1 & 0,1 & neo & naturalized \\
\hline Xanthium albinum & 1 & 0,1 & neo & naturalized \\
\hline Aster lanceolatus & 854 & 72,0 & neo & invasive \\
\hline Bidens frondosa & 593 & 50,0 & neo & invasive \\
\hline Impatiens parviflora & 347 & 29,3 & neo & invasive \\
\hline Conyza canadensis & 309 & 26,1 & neo & invasive \\
\hline Erigeron annuus subsp. annuus & 233 & 19,6 & neo & invasive \\
\hline Echinocystis lobata & 193 & 16,3 & neo & invasive \\
\hline Solidago gigantea & 96 & 8,1 & neo & invasive \\
\hline Arrhenatherum elatius & 65 & 5,5 & neo & invasive \\
\hline Amaranthus retroflexus & 57 & 4,8 & neo & invasive \\
\hline Amaranthus powellii & 44 & 3,7 & neo & invasive \\
\hline Impatiens glandulifera & 32 & 2,7 & neo & invasive \\
\hline Galinsoga parviflora & 27 & 2,3 & neo & invasive \\
\hline Helianthus tuberosus & 24 & 2,0 & neo & invasive \\
\hline Solidago canadensis & 14 & 1,2 & neo & invasive \\
\hline Galinsoga quadriradiata & 8 & 0,7 & neo & invasive \\
\hline Sisymbrium loeselii & 7 & 0,6 & neo & invasive \\
\hline Ambrosia artemisiifolia & 6 & 0,5 & neo & invasive \\
\hline Oxalis dillenii & 6 & 0,5 & neo & invasive \\
\hline Reynoutria sachalinensis & 5 & 0,4 & neo & invasive \\
\hline Rudbeckia laciniata & 2 & 0,2 & neo & invasive \\
\hline
\end{tabular}


Maděra P. et al.: Vascular plant biodiversity of floodplain forest in Morava and Dyje rivers confluence (forest district Soutok), Czech Republic

As regards, specially protected and endangered species, there were 142 of them in the study area (Table 8). 40 species within the total number of 505 records in the segments were protected by law.

Table 8: The presence of endangered herb species (according to Grulich \& Chobot, 2017).

\begin{tabular}{|c|c|c|c|}
\hline species & $\begin{array}{c}\text { number of } \\
\text { segments }\end{array}$ & threat and & ategory \\
\hline Leucojum aestivum & 142 & $\mathrm{C} 1 \mathrm{~b}$ & $\S 1$ \\
\hline Cardamine parviflora & 12 & $\mathrm{C} 1 \mathrm{~b}$ & $\S 1$ \\
\hline Pulicaria dysenterica & 5 & $\mathrm{C} 1 \mathrm{~b}$ & \\
\hline Hierochloë repens & 2 & $\mathrm{C} 1 \mathrm{~b}$ & $\S 1$ \\
\hline Clematis integrifolia & 1 & $\mathrm{C} 1 \mathrm{~b}$ & $\S 1$ \\
\hline Cyperus michelianus & 1 & $\mathrm{C} 1 \mathrm{~b}$ & $\S 1$ \\
\hline Juncus atratus & 1 & $\mathrm{C} 1 \mathrm{~b}$ & $\S 1$ \\
\hline Trapa natans & 1 & $\mathrm{C} 1 \mathrm{~b}$ & $\S 1$ \\
\hline Viola elatior & 56 & $\mathrm{C} 1 \mathrm{t}$ & $\S 1$ \\
\hline Pulegium vulgare & 9 & $\mathrm{C} 1 \mathrm{t}$ & \\
\hline Pulicaria vulgaris & 9 & $\mathrm{C} 1 \mathrm{t}$ & \\
\hline Xanthium strumarium & 7 & $\mathrm{C} 1 \mathrm{t}$ & \\
\hline Lathyrus palustris & 2 & $\mathrm{C} 1 \mathrm{t}$ & $\S 1$ \\
\hline Crepis setosa & 1 & $\mathrm{C} 1 \mathrm{t}$ & \\
\hline Nymphoides peltata & 1 & $\mathrm{C} 1 \mathrm{t}$ & $\S 1$ \\
\hline Scorzonera laciniata & 1 & $\mathrm{C} 1 \mathrm{t}$ & \\
\hline Stratiotes aloides & 1 & $\mathrm{C} 1 \mathrm{t}$ & $\S 2$ \\
\hline Leonurus marrubiastrum & 238 & $\mathrm{C} 2 \mathrm{~b}$ & \\
\hline Scutellaria hastifolia & 93 & $\mathrm{C} 2 \mathrm{~b}$ & $\S 2$ \\
\hline Cicuta virosa & 78 & $\mathrm{C} 2 \mathrm{~b}$ & \\
\hline Verbascum blattaria & 67 & $\mathrm{C} 2 \mathrm{~b}$ & \\
\hline Cnidium dubium & 28 & $\mathrm{C} 2 \mathrm{~b}$ & \\
\hline Sium latifolium & 24 & $\mathrm{C} 2 \mathrm{~b}$ & \\
\hline Euphorbia lucida & 18 & $\mathrm{C} 2 \mathrm{~b}$ & $\S 1$ \\
\hline Thalictrum flavum & 15 & $\mathrm{C} 2 \mathrm{~b}$ & $\S 2$ \\
\hline Lycopus exaltatus & 10 & $\mathrm{C} 2 \mathrm{~b}$ & \\
\hline Iris variegata & 7 & $\mathrm{C} 2 \mathrm{~b}$ & $\S 2$ \\
\hline Teucrium scordium & 6 & $\mathrm{C} 2 \mathrm{~b}$ & $\S 2$ \\
\hline Hydrocharis morsus-ranae & 5 & $\mathrm{C} 2 \mathrm{~b}$ & \\
\hline
\end{tabular}




\begin{tabular}{|c|c|c|c|}
\hline Iris graminea & 5 & $\mathrm{C} 2 \mathrm{~b}$ & $\S 2$ \\
\hline Scirpoides holoschoenus & 5 & $\mathrm{C} 2 \mathrm{~b}$ & \\
\hline Senecio sarracenicus & 5 & $\mathrm{C} 2 \mathrm{~b}$ & $\S 2$ \\
\hline Epipactis albensis & 3 & $\mathrm{C} 2 \mathrm{~b}$ & $\S 2$ \\
\hline Lythrum hyssopifolia & 2 & $\mathrm{C} 2 \mathrm{~b}$ & \\
\hline Lythrum virgatum & 2 & $\mathrm{C} 2 \mathrm{~b}$ & \\
\hline Ophioglossum vulgatum & 2 & $\mathrm{C} 2 \mathrm{~b}$ & $\S 3$ \\
\hline Muscari neglectum & 1 & $\mathrm{C} 2 \mathrm{~b}$ & \\
\hline Ornithogalum boucheanum & 1 & $\mathrm{C} 2 \mathrm{~b}$ & \\
\hline Sonchus palustris & 1 & $\mathrm{C} 2 \mathrm{~b}$ & \\
\hline Stellaria palustris & 1 & $\mathrm{C} 2 \mathrm{~b}$ & \\
\hline Viola tricolor subsp. curtisii & 1 & $\mathrm{C} 2 \mathrm{~b}$ & \\
\hline Carex strigosa & 235 & $\mathrm{C} 2 \mathrm{r}$ & \\
\hline Carex fritschii & 2 & $\mathrm{C} 2 \mathrm{r}$ & \\
\hline Parietaria officinalis & 1 & $\mathrm{C} 2 \mathrm{r}$ & \\
\hline Carex melanostachya & 38 & $\mathrm{C} 2 \mathrm{t}$ & $\S 2$ \\
\hline Althaea officinalis & 11 & $\mathrm{C} 2 \mathrm{t}$ & \\
\hline Gratiola officinalis & 9 & $\mathrm{C} 2 \mathrm{t}$ & $\S 2$ \\
\hline Viola pumila & 3 & $\mathrm{C} 2 \mathrm{t}$ & $\S 2$ \\
\hline Viola stagnina & 2 & $\mathrm{C} 2 \mathrm{t}$ & $\S 2$ \\
\hline Senecio erraticus & 349 & $\mathrm{C} 3$ & \\
\hline Carex divulsa & 264 & $\mathrm{C} 3$ & \\
\hline Cardamine dentata & 236 & $\mathrm{C} 3$ & \\
\hline Barbarea stricta & 178 & $\mathrm{C} 3$ & \\
\hline Lotus tenuis & 56 & $\mathrm{C} 3$ & \\
\hline Cucubalus baccifer & 45 & $\mathrm{C} 3$ & \\
\hline Euphorbia palustris & 40 & $\mathrm{C} 3$ & $\S 2$ \\
\hline Pseudolysimachion maritimum & 37 & $\mathrm{C} 3$ & \\
\hline Corydalis pumila & 32 & $\mathrm{C} 3$ & \\
\hline Carex curvata & 30 & $\mathrm{C} 3$ & \\
\hline Silaum silaus & 30 & $\mathrm{C} 3$ & \\
\hline Dipsacus laciniatus & 26 & $\mathrm{C} 3$ & \\
\hline Erysimum diffusum & 21 & $\mathrm{C} 3$ & \\
\hline Galanthus nivalis & 15 & $\mathrm{C} 3$ & $\S 3$ \\
\hline Leersia oryzoides & 14 & $\mathrm{C} 3$ & \\
\hline Trifolium fragiferum var. fragiferum & 13 & $\mathrm{C} 3$ & \\
\hline
\end{tabular}


Maděra P. et al.: Vascular plant biodiversity of floodplain forest in Morava and Dyje rivers confluence (forest district Soutok), Czech Republic

\begin{tabular}{|c|c|c|c|}
\hline Verbena officinalis & 11 & $\mathrm{C} 3$ & \\
\hline Hottonia palustris & 11 & $\mathrm{C} 3$ & $\S 3$ \\
\hline Carex distans & 10 & $\mathrm{C} 3$ & \\
\hline Gagea minima & 10 & $\mathrm{C} 3$ & \\
\hline Thalictrum lucidum & 10 & $\mathrm{C} 3$ & \\
\hline Bromus commutatus & 8 & $\mathrm{C} 3$ & \\
\hline Achillea pannonica & 8 & $\mathrm{C} 3$ & \\
\hline Myosurus minimus & 8 & $\mathrm{C} 3$ & \\
\hline Centaurium pulchellum & 6 & $\mathrm{C} 3$ & \\
\hline Iris sibirica & 6 & $\mathrm{C} 3$ & $\S 2$ \\
\hline Linaria genistifolia & 5 & $\mathrm{C} 3$ & \\
\hline Scilla vindobonensis & 5 & $\mathrm{C} 3$ & $\S 2$ \\
\hline Chondrilla juncea & 4 & $\mathrm{C} 3$ & \\
\hline Cyperus fuscus & 3 & $\mathrm{C} 3$ & \\
\hline Allium angulosum & 2 & $\mathrm{C} 3$ & $\S 2$ \\
\hline Hesperis sylvestris & 2 & $\mathrm{C} 3$ & \\
\hline Hyoscyamus niger & 1 & $\mathrm{C} 3$ & \\
\hline Carex supina & 1 & $\mathrm{C} 3$ & \\
\hline Ficaria calthifolia & 1 & $\mathrm{C} 3$ & \\
\hline Gagea pusilla & 1 & $\mathrm{C} 3$ & \\
\hline Lactuca quercina & 1 & $\mathrm{C} 3$ & \\
\hline Lathyrus latifolius & 1 & $\mathrm{C} 3$ & \\
\hline Muscari comosum & 1 & $\mathrm{C} 3$ & \\
\hline Najas marina & 1 & $\mathrm{C} 3$ & \\
\hline Silene otites & 1 & $\mathrm{C} 3$ & \\
\hline Veronica catenata & 1 & $\mathrm{C} 3$ & \\
\hline Carex riparia & 908 & $\mathrm{C} 4 \mathrm{a}$ & \\
\hline Aristolochia clematitis & 530 & $\mathrm{C} 4 \mathrm{a}$ & \\
\hline Veronica hederifolia & 426 & $\mathrm{C} 4 \mathrm{~b}$ & \\
\hline Galega officinalis & 178 & $\mathrm{C} 4 \mathrm{a}$ & \\
\hline Veronica montana & 123 & $\mathrm{C} 4 \mathrm{a}$ & \\
\hline Cerastium lucorum & 92 & $\mathrm{C} 4 \mathrm{a}$ & \\
\hline Allium ursinum & 89 & $\mathrm{C} 4 \mathrm{a}$ & \\
\hline Myosotis sparsiflora & 71 & $\mathrm{C} 4 \mathrm{a}$ & \\
\hline Galium rivale & 64 & $\mathrm{C} 4 \mathrm{a}$ & \\
\hline Carex buekii & 58 & $\mathrm{C} 4 \mathrm{a}$ & \\
\hline
\end{tabular}




\begin{tabular}{|c|c|c|}
\hline Cardamine matthioli & 55 & $\mathrm{C} 4 \mathrm{a}$ \\
\hline Serratula tinctoria & 31 & $\mathrm{C} 4 \mathrm{a}$ \\
\hline Aethusa cynapioides & 29 & $\mathrm{C} 4 \mathrm{a}$ \\
\hline Nuphar lutea & 20 & $\mathrm{C} 4 \mathrm{a}$ \\
\hline Vicia dumetorum & 20 & $\mathrm{C} 4 \mathrm{a}$ \\
\hline Bromus japonicus & 16 & $\mathrm{C} 4 \mathrm{a}$ \\
\hline Veronica scutellata & 14 & $\mathrm{C} 4 \mathrm{a}$ \\
\hline Dianthus armeria & 13 & $\mathrm{C} 4 \mathrm{a}$ \\
\hline $\begin{array}{l}\text { Verbascum chaixii subsp. } \\
\text { austriacum }\end{array}$ & 13 & $\mathrm{C} 4 \mathrm{a}$ \\
\hline Galium mollugo & 11 & $\mathrm{C} 4 \mathrm{~b}$ \\
\hline Batrachium aquatile & 10 & $\mathrm{C} 4 \mathrm{~b}$ \\
\hline Carex otrubae & 8 & $\mathrm{C} 4 \mathrm{a}$ \\
\hline Centaurium erythraea & 8 & $\mathrm{C} 4 \mathrm{a}$ \\
\hline Peucedanum oreoselinum & 8 & $\mathrm{C} 4 \mathrm{a}$ \\
\hline Berula erecta & 7 & $\mathrm{C} 4 \mathrm{a}$ \\
\hline Petrorhagia prolifera & 7 & $\mathrm{C} 4 \mathrm{a}$ \\
\hline Butomus umbellatus & 6 & $\mathrm{C} 4 \mathrm{a}$ \\
\hline Inula salicina & 6 & $\mathrm{C} 4 \mathrm{a}$ \\
\hline Melica transsilvanica & 6 & $\mathrm{C} 4 \mathrm{a}$ \\
\hline Isopyrum thalictroides & 4 & $\mathrm{C} 4 \mathrm{a}$ \\
\hline Lavatera thuringiaca & 4 & $\mathrm{C} 4 \mathrm{a}$ \\
\hline Cynodon dactylon & 3 & $\mathrm{C} 4 \mathrm{a}$ \\
\hline Bolboschoenus sp. indet. & 3 & $\mathrm{C} 4 \mathrm{a}$ \\
\hline Galium elongatum & 3 & $\mathrm{C} 4 \mathrm{a}$ \\
\hline Geranium sanguineum & 3 & $\mathrm{C} 4 \mathrm{a}$ \\
\hline Malva alcea & 3 & $\mathrm{C} 4 \mathrm{a}$ \\
\hline Primula veris & 3 & $\mathrm{C} 4 \mathrm{a}$ \\
\hline Pseudolysimachion spicatum & 3 & $\mathrm{C} 4 \mathrm{a}$ \\
\hline Scrophularia umbrosa & 3 & $\mathrm{C} 4 \mathrm{a}$ \\
\hline Corydalis intermedia & 2 & $\mathrm{C} 4 \mathrm{a}$ \\
\hline Corynephorus canescens & 2 & $\mathrm{C} 4 \mathrm{a}$ \\
\hline Omphalodes scorpioides & 2 & $\mathrm{C} 4 \mathrm{a}$ \\
\hline Euphorbia esula subsp. riparia & 2 & $\mathrm{C} 4 \mathrm{~b}$ \\
\hline Cerinthe minor & 1 & $\mathrm{C} 4 \mathrm{a}$ \\
\hline Anthericum ramosum & 1 & $\mathrm{C} 4 \mathrm{a}$ \\
\hline Dianthus pontederae & 1 & $\mathrm{C} 4 \mathrm{a}$ \\
\hline
\end{tabular}


Maděra P. et al.: Vascular plant biodiversity of floodplain forest in Morava and Dyje rivers confluence (forest district Soutok), Czech Republic

\begin{tabular}{|l|c|c|l|} 
Listera ovata & 1 & $\mathrm{C} 4 \mathrm{a}$ & \\
\hline Neottia nidus-avis & 1 & $\mathrm{C} 4 \mathrm{a}$ & \\
\hline Polystichum aculeatum & 1 & $\mathrm{C} 4 \mathrm{a}$ & \\
\hline Schoenoplectus lacustris & 1 & $\mathrm{C} 4 \mathrm{a}$ & \\
\hline Viola mirabilis & 1 & $\mathrm{C} 4 \mathrm{a}$ & \\
\hline
\end{tabular}

\section{DISCUSSION}

Floodplain forests often represent a high biodiversity area in the European landscape (Ward et al., 2002). The species richness of certain organisms, such as vascular plants, often far exceeds that in adjacent upland habitats (Naiman et al., 1993; Tabacchi et al., 1996; Stohlgren et al. 1998). Concerning to the vascular plants, the study area isn't exception, we found 761 species (591 excluding adventive species) in area of $51 \mathrm{~km}^{2}$ what corresponds to about a fifth of the flora of the Czech Republic. Many authors confirm the high importance of floodplain forests for vascular plant species diversity maintenance (Tab. 9). Schnitzler et al. (2007) summarised available articles focused on the diversity of riparian forests across the whole of Europe and recorded 1,380 species.

Table 9: Overview of studies focusing on vascular plant diversity of floodplain forests

\begin{tabular}{|c|c|c|c|}
\hline Locality & Number of species & Size of area & Source \\
\hline $\begin{array}{l}\text { Forest district Valtice, Thaya River, } \\
\text { Czech Republic }\end{array}$ & 656 & $16 \mathrm{~km}^{2}$ & Maděra et al. (2011) \\
\hline $\begin{array}{l}\text { Forest district Tvrdonice, Morava } \\
\text { River, Czech Republic }\end{array}$ & 612 & $22 \mathrm{~km}^{2}$ & Maděra et al. (2013) \\
\hline northern Croatia & 437 & & Trinajstič et al. (2005) \\
\hline $\begin{array}{l}\text { Current and Jacks Fork Rivers, } \\
\text { North America }\end{array}$ & 269 & & Lyon \& Sagers (1998) \\
\hline Adour River (SW France) & 1,396 & & Tabacchi et al. (1996) \\
\hline Pantanal wetland, Brazil & 2000 & $150,000 \mathrm{~km}^{2}$ & Pott et al. (2011) \\
\hline Seine, France & 334 & 20 plots $1 \mathrm{~km}^{2}$ & Ernoult et al. (2006) \\
\hline NE Wisconsin, USA & 162 & 417 plots $1 \mathrm{~m}^{2}$ & Goebel et al. (2006) \\
\hline Danube River & 165 & & Mölder et al. (2011) \\
\hline Sado and Guadiana, Portugal & 45 (only woody plants) & 70 river sections of $2 \mathrm{~km}$ & Santos (2010) \\
\hline Estonia & 372 & 1600 plots $1 \mathrm{~m}^{2}$ & Paal et al. (2007) \\
\hline $\begin{array}{l}\text { Current River and Jacks Fork River, } \\
\text { SW Missouri, USA }\end{array}$ & 339 & 94 plots & Lyon \& Sagers (1998) \\
\hline Cypress Creek NWR, Illinois, USA & 193 & 80 plots $1 \mathrm{~m}^{2}$ & McLane et al. (2012) \\
\hline Ill, Rhine, Loire and Allier & $106-157$ & & Schnitzler (1997) \\
\hline Rhine & 37 (only woody plants) & & Trémoliéres et al. (1998) \\
\hline
\end{tabular}

Řepka at al. (2015) recorded a total of 732 herb and 121 woody species in whole area $\left(89 \mathrm{~km}^{2}\right)$ of the floodplain forests of Forest Enterprise Židlochovice (Forest Districts Tvrdonice, Valtice and Soutok) demonstrating their immense importance for biodiversity of vascular plants.

History of forest management in the study area is crucial for understanding of the highly valuable current state. In the Middle Ages, coppice forests with 7 year rotation are described and coppices with standard are documented, too (Nožička, 1956). The forests were used for 
livestock grazing (especially pigs) due to acorn production. The "modern forest management" began under the Lichtenstein family ownership in the middle of the $18^{\text {th }}$ century (Hrib, 2004). The conversion of coppice forest to high forest started by using the way of alternate forestry (agroforestry) system. Man-made natural ecosystems sensu van Maarel (1975) were established this way, forests developed under influence of both, human activities and natural processes. Key human interventions supporting the high level of biodiversity are (i) using of habitat-original tree species (mainly oak, ash and elm) for reforestation, especially oak regeneration is problematic without artificial reforestation (Libus et al., 2010)

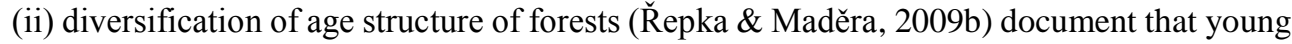
developmental stages of forests host high diversity), (iii) creation of forest edges as a habitat with high diversity and many endangered plant species (Maděra et al., 2011), (iv) maintenance of water channels bringing water inside the floodplain forests after rivers have been regulated (Vybíral \& Hrib, 2000). The most important natural conditions co-creating the floodplain forests are (i) meandering rivers, (ii) high groundwater table and nutrient reach fluvisols and (iii) regular flooding (Klimo et al., 2008).

Human modifications of streams and rivers have caused extensive stream channel and riparian degradation (Meixler \& Bain, 2010). Since 1973, the south Moravian floodplain forests were affected by Dyje and Morava Rivers regulation within complex hydrotechnical measures (Jakubec, 1981). The most serious problem of the study area is the absence of natural hydrological regime due to the regulation of main water courses since this time. The variety of riparian plant communities found in natural floodplains is mainly controlled by the flow regime (Poff et al., 1997), which generates physical disturbance and environmental stress on riparian vegetation, ultimately affecting its temporal and spatial dynamics (Shafroth et al., 2002). The dynamic fluvial succession by the absence of natural hydrological regime lead to the increase of occurrence of late-serial stages (the driest types of hard-wood forests) and on the contrary, to the decrease of initial-serial stages. Gonzáles (2010) described progressive area decrease (up to $37 \%$ ) of the pioneer forest types (Populus nigra, Salix alba and Tamarix spp.) since the intensification of river regulation in the mediterranean region. In contrast, non-pioneer senescent forests have doubled their surface after river regulation was intensified. The same results were published by Maděra et al. (2010) from area under study, $60 \%$ of area was occupied by "wet hardwood" floodplain forests communities (Querci roboris-Fraxineta) and $30 \%$ of area was occupied by "dry hardwood" floodplain forests communities (Ulmi-Fraxineta carpini) before rivers regulations. The rate was opposite after 30 years of development without flooding and decreasing ground water table (Penka et al., 1991). Dams, land-use changes throughout the basin, and construction of flood defences that restrict the main channel have changed behaviour of the Ebro river system which urgently needs a management plan combining both, improvement and risk reduction (Ollero, 2010).

Technical regulations of the water regime within floodplains can also impact on the species diversity of floodplain forests. For example, Trémoliéres et al. (1998) compared various sections of an alluvial hardwood forest along the Rhine. Using six plots of about $2,000 \mathrm{~m}^{2}$, they found 63 species ( 25 woody species) in a flooded floodplain, 121 species (45 woody species) in a floodplain that had not been flooded for 30 years, and 95 species (47 woody species) in a floodplain not flooded for 130 years. Deiller et al. (2001) mentioned that the species richness of the extant vegetation increases with the duration of interruption of the floods in the Rhine forest as a result of introduction of flood-intolerant species in the unflooded forest. By contrast, Amanda et al. (2005) recorded a $40 \%$ higher number of species in unregulated floodplain of the Yampa river in contrast to the regulated Green River. Other authors also document the changes in species composition and spatial structure of the synusia of floodplain forest herb layer (Vašíček, 1985; Vrška, 1997, 1998; Maděra 2001a, 
Maděra P. et al.: Vascular plant biodiversity of floodplain forest in Morava and Dyje rivers confluence (forest district Soutok), Czech Republic

2001b; Viewegh, 2002; Unar \& Šamonil, 2008; Santos, 2010) or in the tree layer (Schnitzler 1994; Trémoliéres et al., 1998; Janík et al., 2008, 2011, 2016) in dependence on drying of floodplain forests, when flood-intolerant and mesic species can arrive.

High native plant diversity in riparian biotopes is largely associated with natural disturbance, particularly flooding and scour by seasonal and storm related flood pulses, which create regeneration microsites and mediate resource competition among species (Naiman \& Decamps, 1997; Naiman et al., 1993, 2005). Frequent natural or anthropogenic disturbances, however, can also create conditions conducive to alien plant establishment (De Ferrari \& Naiman, 1994; Pyšek \& Prach, 1994; Planty-Tabacchi et al., 1996; Pyle, 1995; Stohlgren et al., 1998). We found 177 adventive species in the study area, it is $23.2 \%$ of all vascular plants creating the floodplain forest communities. Many other authors confirmed the sensitivity of floodplain forests to adventive species invasion (Tab 10).

Table 10 Comparison of adventive vascular plant species occurrence in floodplain forests in various parts of the World

\begin{tabular}{|l|c|c|c|}
\hline Locality & $\begin{array}{c}\text { No of adventive } \\
\text { species }\end{array}$ & $\begin{array}{c}\text { Ratio of the } \\
\text { total }(\%)\end{array}$ & Source \\
\hline $\begin{array}{l}\text { Allegheny River Islands Wilderness } \\
\text { (northwestern Pennsylvania) }\end{array}$ & 40 & 17.8 & Williams (2010) \\
\hline Mura River (NE Slovenia) & & 15.0 & Košir et al. (2013) \\
\hline Cypress Creek NWR, Illinois, USA & & 14.4 & McLane et al. $(2012)$ \\
\hline $\begin{array}{l}\text { Yampa and Green rivers (northwest } \\
\text { Colorado, USA) }\end{array}$ & & 30.0 & Amanda et al. (2005) \\
\hline Upper Danube & & 7.0 & Mölder \& Schneider 2011 \\
\hline Middle Danube & & 14.0 & Mölder \& Schneider 2011 \\
\hline Lower Danube & & 10.0 & Mölder \& Schneider 2011 \\
\hline eastern Oregon, USA & 60 & 14.5 & Magee et al. $(2008)$ \\
\hline
\end{tabular}

Schnitzler et al. (2007) summarised 1,380 species across European riparian forests, 45 (3.3\%) of these were exotic (adventive) species. Pyšek et al. (2012b) found that the proportion of neophytes in floodplain forests of the alliance Alnion incanae (incl. Ulmenion suballiance) was $2.2 \pm 2.8 \%$, in coverage $4 \pm 10 \%$, which is the highest number within the forest communities of the Czech Republic. Our study shows higher average proportion of neophytes in the study area $(10.5 \%)$. A number of large-scale studies have confirmed that floodplain forests are one of the most invaded forest habitats (Chytrý et al., 2005; Petrášová et al., 2013; К̌epka et al., 2015).

Many exotics found in Schnitzler's et al (2007) study were introduced intentionally either from North America (51\%) or Asia (38\%). The exotics belong to various life-forms: approximately $50 \%$ are grasses (polycarpic perennials, summer and autumn annuals), while the rest are phanerophytes, equally distributed among trees, shrubs and liana life-forms. Most of the exotics are thermophilous and light-demanding pioneer species from warm temperate floodplains. Thirty-two percent are from the Asteraceae family. The distribution of exotics in the 177 communities recorded is highly unequal. Twenty-six are present at low levels in very few communities; seven have an intermediate distribution; and twelve (27\%) are abundant in a large range of habitats (in compliance with our results there are for example Impatiens parviflora, Erigeron canadensis or Solidago gigantea). The most important adventive tree species were Fraxinus pennsylvanica (Middle and Lower Danube), Acer negundo (Middle Danube) and Robinia pseudacacia (Upper Danube), which reached considerable proportions in the tree layer. Frequent adventive herb species were Impatiens parviflora and Solidago 
gigantea (Upper and Middle Danube), Aster parviflorus, Oxalis stricta (Middle Danube) and Aster lanceolatus (Lower Danube). The invasive shrub species Amorpha fruticosa was very common on the Lower Danube (Mölder \& Schneider, 2011). Also, Chmura \& Sierka (2006) in their study of Polish floodplain forests consider Impatiens parviflora to be a significant invasive species.

Floodplains are considered vulnerable to exotic species (Hood \& Naiman, 2000; Harris et al., 2005), due to the combined influence of intensive human exploitation, a high degree of hydrological connectivity that facilitates propagule dispersal and the high spatial and temporal heterogeneity inherent to these systems. Globally, anthropogenic alterations to floodplain hydrological regimes have frequently resulted in riparian species invasions (Richardson et al., 2007). Vegetation changes are partially structured by reduced flood frequency favouring increased abundance of exotic, sexually reproducing annuals at drier sites. Sites of low flood frequency are more sensitive to future exotic weed invasion. Flow restoration is predicted to benefit propagule dispersal of species adopting dual regeneration strategies, which are predominantly natives in this system (Stokes et al., 2010). The invasion by alien plant species is a major challenge to the conservation and management of riparian areas, which can alter ecosystem structure and function in undesirable ways (Hood \& Naiman, 2000; Stohlgren et al., 1998). The invasive species capable of becoming dominant are the most dangerous, and in the study area it is Aster lanceolatus (̌̌epka et al., 2009a). Brewer (2010) described a similar example: a significant negative effect of species richness on invasive grass Microstegium vimineum abundance. According to investigation of Saccone et al. (2010), Acer negundo showed both a high survival in the shade and a high growth in full light. This species could be an example of adaptive plasticity that certainly represents a competitive advantage over native species. Another example is mentioned by Hanula \& Horn (2011); they investigated the effects of the invasive shrub Chinese privet (Ligustrum sinense). Pyšek \& Prach (1993) named four significant invasive species in riparian habitats of central Europe: Impatiens glandulifera, Heracleum mantegazzianum, Reynoutria japonica and $R$. sachalinensis; none of these has caused a significant problem in the study area.

\section{CONCLUSIONS}

Natural riparian corridors are the most diverse, dynamic and complex biophysical habitats on the terrestrial part of the Earth. Riparian corridors, as interfaces between terrestrial and aquatic systems, encompass sharp environmental gradients, ecological processes and communities. Riparian corridors are an unusually diverse mosaic of landforms, communities and environments within wider landscape. They serve as a framework for understanding of the organisation, diversity and dynamics of communities associated with fluvial ecosystems. Riparian corridors possess an unusually diverse array of species and environmental processes and they should play an essential role in water and landscape planning, in the restoration of aquatic systems, and in catalyzing institutional and societal cooperation for these efforts (Naiman et al., 1993).

Unfortunately, floodplains forests belong to the most endangered communities not only in Europe (Wenger et al., 1990; Klimo \& Hager, 2000) affected by diverse human negative interventions. The most serious are hydrotechnical river regulations (Dynesius \& Nilsson, 1994). Meixler \& Bain (2010) developed the cost-effective, rapid assessment tools can be used to better manage such areas by identifying the status of habitats for restoration planning and protection. Managers can use these cost-effective strategy development tools to identify candidate reaches for further study and prioritize stream channel and riparian restoration 
Maděra P. et al.: Vascular plant biodiversity of floodplain forest in Morava and Dyje rivers confluence (forest district Soutok), Czech Republic

actions over large regions. Our results could serve as an unique basis for such management measures.

Gonzáles (2010) recommended measures principally aimed at recovering some hydrogeomorphic dynamism to guarantee the self-sustainability of the floodplain forest ecosystem. In this sense, according to Comín et al. (2005), the most effective restoration approach should focus on the recovery of some hydrogeomorphic dynamism (i.e., channel migration, periodic creation of new barren sites, reactivation of secondary channels, meander cut-offs, renaturalized hydroperiod, etc.) both, at the basin and the reach scale, within the current socioeconomic context. Thus, the ideal hydrogeomorphic regime would not necessarily be the preregulation state but one 'renaturalized', which led to a selfsustainable forest structure at patch and landscape scale, guaranteed their ecological functions and provided services to society (Dufour \& Piégay, 2009).

\section{ACKNOWLEDGEMENT}

The results could be attained thanks to support provided by NAZV (National Agency for Research in Agriculture) project called Harmonization of Forest Economy in Floodplains as a Tool to Preserve Species Diversity of Vascular Plants (reg. no. QI92A031).

\section{REFERENCES}

Amanda, L., Uowolo A.L., Binkley D., Adair E.C. (2005). Plant diversity in riparian forests in northwest Colorado: Effects of time and river regulation. Forest Ecology and Management 218: 107-114.

Brewer, J.S. (2010). A Potential Conflict between Preserving Regional Plant Diversity and Biotic Resistance to an Invasive Grass, Microstegium vimineum. Natural Areas Journal, 30 (3): 279-293.

Chmura, D., Sierka, E. (2006). Relation between invasive plant and species richness of forest floor vegetation: A study of Impatiens parviflora DC. Polish Journal of Ecology, 54 (3): 417-428.

Chytrý, M. (ed.) (2013). Vegetace České republiky. 4. Lesní a křovinná vegetace. Academia, Praha, $552 \mathrm{p}$.

Chytrý, M., Pyšek, P., Tichý, L., Knollová, I., Danihelka, J. (2005). Invasions by alien plants in the Czech Republic: a quantitative assessment across habitats. Preslia 77: 339-354.

Comín, F.A., Menéndez, M., Pedrocchi, C., Moreno, S., Sorando, R., Cabezas, A., García, M., Rosas, V., Moreno, D., González, E., Gallardo, B., Herrera, J.A., Ciancarelli, C. (2005). Wetland restoration: integrating scientific-technical, economic, and social perspectives. Ecological Restoration 23(3):182-186.

Danihelka, J., Grulich, V., Šumberová, K., Řepka, R., Husák, Š. et Čáp, J. (1995). O rozšíření některých cévnatých rostlin na nejjižnější Moravě. Zprávy České Botanické Společnosti, 30 (Suppl.): 29-102.

Danihelka, J., Šumberová, K. (2004). O rozšǐření některých cévnatých rostlin na nejjižnější Moravě II. Př́roda, 21: 117-192, Praha. 
De Ferrari, C. M., Naiman, R. J. (1994). A multi-scale assessment of the occurrence of exotic plants on the Olympic Peninsula, Washington. Journal of Vegetation Science 5: 247258.

Deiller, A.F., Walter, J.M.N., Trémoliéres, M. (2001). Effect of flood interruption on species richness, diversity and floristic composition of woody regeneration in the upper Rhine alluvial hardwood forest. Regulated Rivers: Research \& Management, 17: 393-405.

Dufour, S., Piégay, H. (2009). From the myth of a lost paradise to targeted river restoration: forget natural references and focus on human benefits. River Research and Applications 25 (5): 568-581.

Dynesius, M., Nilsson, C. (1994). Fragmentation and flow regulation of river systems in the northern third of the world. Science 266: 753-761.

Ernoult, A., Tremauville, Y., Cellier, D., Margerie, P., Langlois, E., Alard, D. (2006). Potential landscape drivers of biodiversity components in a flood plain: Past or present patterns? Biological Conservation, 127: 1-17.

Goebel, P.CH., Pregitzer, K.S., Palik, B.J. (2006). Landscape hierarchies influence riparian ground-flora communities in Wisconsin, USA. Forest Ecology and Management 230: 43-54 González, E., González-Sanchis, M., Cabezas, A., Comín, F.A., Muller, E. (2010). Recent Changes in the Riparian Forest of a Large Regulated Mediterranean River: Implications for Management. Environmental Management 45: 669-681.

Grulich, V., Chobot, K. (eds.) (2017). Červený seznam ohrožených druhů České republiky. Cévnaté rostliny. Praha, Agentura ochrany prŕrody a krajiny ČR.

Hanula, J.L., Horn, S. (2011). Removing an exotic shrub from riparian forests increases butterfly abundance and diversity. Forest Ecology and Management 262: 674-680.

Harris, M.B., Tomas, W., Mourao, G., Da Silva, C.J., Guimaraes, E., Sonoda, F., Fachim, E. (2005). Safeguarding the Patanal wetlands: threats and conservation initiatives. Conservation Biology 19: 714-720.

Hood, G.W., Naiman, R.J. (2000). Vulnerability of riparian zones to invasion by exotic vascular plant species. Plant Ecology, 148: 105-114.

Horák, J. (1961). Jihomoravské lužní lesy (typologická studie). Thesis, VŠZ, Brno, 266 pp.

Hrib, M. (2004). Z historie lesního hospodářství. In. Hrib M., Kordiovský E. (eds.): Lužní les v Dyjsko-moravské nivě (pp. 209-226). Moraviapress, Břeclav.

Hrib, M., Kordiovský, E. (eds.) (2004). Lužní les v Dyjsko-moravské nivě. Moraviapress, Břeclav, 591 p.

Jakubec, B. (1981). Vodohospodářské úpravy na jižní Moravě. Lesnická práce, 5: 204-212.

Janík, D., Adam, D., Vrška, T., Hort, L., Unar, P., Král, K., Šamonil, P., Horal, D. (2008). Tree layer dynamics of the Cahnov-Soutok near-natural floodplain forest after 33 years (1973-2006). European Journal of Forest Research, 127 (4): 337-345.

Janík, D., Adam, D., Vrška, T., Hort, L., Unar, P., Král, K., Šamonil, P., Horal, D. (2011). Field maple and hornbeam populations along a 4-m elevation gradient in an alluvial forest. European Journal of Forest Research, 130: 197-208.

Janík, D., Adam, D., Hort, L., Král, K., Šamonil, P., Unar, P., Vrška, T. (2016). Patterns of Fraxinus angustifolia in an alluvial old-growth forest after declines in flooding events. European Journal of Forest Research, 135: 215-228.

Klimo, E., Hager, H. (2000). The floodplain forests in Europe. EFI, Leiden, Boston, Köln, Brill, 215 p. 
Maděra P. et al.: Vascular plant biodiversity of floodplain forest in Morava and Dyje rivers confluence (forest district Soutok), Czech Republic

Klimo, E., Hager, H., Matić, S., Anić, I., Kulhavý, J. /eds./ (2008). Floodplain Forests of the Temperate Zone of Europe. Lesnická práce, Kostelec nad Černými Lesy, 623 p.

Košir, P., Čarni, A., Marinšek, A., Šilc, U. (2013). Floodplain forest communities along the Mura River (NE Slovenia). Acta Botanica Croatia, 72, 1: 71-95.

Kubát, K., Hrouda, L., Chrtek, J., Kaplan, Z., Kirschner, J., Štěpánek, J. (2002). Klíč ke květeně České republiky. Academia, Praha, 928 p.

Laštůvka, Z., Barták, M., Bezděk, J., Bílý, S., Čelechovský, A., Dolný, A., Hula, V., Chládek, F., Ježek, J., Kment, P., Malenovský, I., Řezníčková, P., Ř́ha, M., Skuhravá, M., Stejskal, R., Šefrová, H., Tkoč, M., Trnka, F., Vašátko, J. (2016). Červená kniha ohrožených druhů bezobratlých lužnich lesů Biosférické rezervace Dolní Morava. Mendel University, Brno, $260 \mathrm{p}$.

Libus, J., Mauer, O., Vavř́íček, D. (2010). Soil preparation by ploughing in the floodplain forest and its influence on vegetation and primary soil characteristics. Journal of Forest Science, 56 (4): 183-194.

Lyon, J., Sagers, C.L. (1998). Structure of herbaceous plant assemblages in a forested riparian landscape. Plant Ecology 138: 1-16.

Nožička, J. (1956): Z minulosti jihomoravských luhů (Předběžná studie). Práce výzkumných ústavů lesnických ČSR, sv.10: 169-199.

Maarel, van der, E. (1975). Man-made natural ecosystems in environmental management and planning. In: Dobben van W.H., Lowe-McConell R.H. (eds.): Unifying concepts in ecology (pp. 263-274). The Hague, Dr W. Junk B. V. Publishers.

Machar, I., Čermák, P., Pechanec, V. (2018). Ungulate Browsing Limits Bird Diversity of the Central European Hardwood Floodplain Forests. Forests, 9: 373; doi:10.3390/f9070373.

Maděra, P. (2001a). Response of floodplain forest communities herb layer to changes in the water regime. Biológia, 56: 63-72, Bratislava.

Maděra, P. (2001b). Effect of water regime changes on the diversity of plant communities in floodplain forests. Ekológia, 20 (Supplement 1): 116-129, (Bratislava).

Maděra, P. et al. (2007). 100 nejzajímavějších stromů Biosférické rezervace Dolní Morava. BR Dolní Morava, 120 p.

Maděra, P., Vahalík, P., Řepka, R., Mikita, T. (2010). Odraz změn vodního režimu lužních lesů LZ Židlochovice v lesnické typologii (Atlas změn skupin typů geobiocénů v letech 1964 a 2001). Lesnická práce, Kostelec nad Černými Lesy, 20 p. + 8 maps 1:20 000.

Maděra, P., Šebesta, J., Řepka, R., Klimánek, M. (2011). Vascular plants distribution as a tool for adaptive forest management of floodplain forests in the Dyje river basin. Journal of Landscape Ecology, 4, 2: 18-34.

Maděra, P., Řepka, R., Šebesta, J., Koutecký, T., Klimánek, M. (2013). Vascular plant biodiversity of floodplain forest geobiocoenosis in Lower Morava river basin (forest district Tvrdonice), Czech Republic. Journal of Landscape Ecology, 6, 2: 34-64.

Magee, T.K., Ringold, P.L., Bollman, M.A. (2008). Alien species importance in native vegetation along wadeable streams, John Day River basin, Oregon, USA. Plant Ecology 195: 287-307.

McLane, C.R., Battaglia, L.L., Gibson, D.J., Groninger, J.W. (2012). Succession of Exotic and Native Species Assemblages within Restored Floodplain Forests: A Test of the Parallel Dynamics Hypothesis. Restoration Ecology 20 (2): 202-210. 
Meixler, M.S., Bain, M.B. (2010). Landscape scale assessment of stream channel and riparian habitat restoration needs. Landscape Ecological Engineering 6: 235-245.

Miklín, J., Čížek, L. (2014). Erasing a European biodiversity hot-spot: Open woodlands, veteran trees and mature forests succumb to forestry intensification, succession, and logging in a UNESCO Biosphere Reserve. Journal for Nature Conservation, 22: 35-41.

Miklín, J, Sebek, P, Hauck, D, Konvicka, O, Cizek, L. (2018). Past levels of canopy closure affect the occurrence of veteran trees and flagship saproxylic beetles. Diversity and Distributions, 24: 208-218.

Miklín, J., Hauck, D., Konvička, O., Cizek, L. (2017). Veteran trees and saproxylic insects in the floodplains of lower Morava and Dyje rivers, Czech Republic. Journal of Maps, 13, 291 299.

Mölder, A., Schneider, E. (2011). On the beautiful diverse Danube? Danubian floodplain forest vegetation and flora under the influence of river eutrophication. River Research and Applications, 27: 881-894.

Naiman, R.J., Decamps, H., Pollock, M. (1993). The role of riparian corridors in maintaining regional biodiversity. Ecological Applications, 3: 209-212.

Naiman, R. J., Decamps, H. (1997). The ecology of interfaces: Riparian zones. Annual Review of Ecology, Evolution and Systematics, 28: 621-658.

Naiman, R. J., Decamps, H., MC Clain, M.E. (2005). Riparia: Ecology, Conservation, and Management of Streamside Communities. Elsevier Academic Press, New York.

Ollero, A. (2010). Channel changes and floodplain management in the meandering middle Ebro River, Spain. Geomorphology 117: 247-260.

Opluštil, L., Čupa, P. (2012). Owls of the Lower Morava Biosphere Reserve. Biosférická rezervace Dolní Morava, $26 \mathrm{p}$.

Paal, J., Rannik, R., Jeletsky, E.M., Prieditis, N. (2007). Floodplain forests in Estonia: Typological diversity and growth conditions. Folia Geobotanica 42: 383-400.

Penka, M., Vyskot, M., Klimo, E., Vašíček, F. (1991). Floodplain forest ecosystem 2. Academia, Praha, 632 p.

Petrášová, M., Jarolímek, I., Medvecká, J. (2013). Neophytes in Pannonian hardwood floodplain forests - History, present situation and trends. Forest Ecology and Management 308: 31-39.

Planty-Tabacchi, A., Tabacchi, E., Naiman, R. J., De Ferrari, C. M., Decamps, H. (1996). Invasibility of species-rich communities in riparian zones. Conservation Biology, 10: 598607.

Poff, N.L., Allan, J.D., Bain, M.B., Karr, J.R., Prestegaard, K.L., Richter, B.D., Sparks, R.E., Stromberg, J.C. (1997). The natural flow regime. A paradigm for river conservation and restoration. Bioscience 47: 769-784.

Pott, A., Oliveira, A.K.M., Damasceno-Junior, G.A., Silva, J.S.V. (2011). Plant diversity of the Pantanal wetland. Brazilian Journal of Biology, 71, 1 (suppl.): 265-273.

Pyle, L. L. (1995). Effects of disturbance on herbaceous exotic plant species on the floodplain of the Potomac River. American Midland Naturalist, 134: 244-253.

Pyšek, P., Prach, K. (1993): Plant invasions and the role of riparian habitats: a comparison of four species alien to central Europe. Journal of Biology, 20: 413-420.

Pyšek, P., Prach, K. (1994): How Important are Rivers for Supporting Plant Invasions? In. L. C. de Waal, L. E. Child, P. M. Wade and J. H. Brock (Eds.): Ecology and Management of 
Maděra P. et al.: Vascular plant biodiversity of floodplain forest in Morava and Dyje rivers confluence (forest district Soutok), Czech Republic

Invasive Riverside Plants (pp. 19-26). John Wiley \& Sons Ltd.

Pyšek, P., Danihelka, J., Sádlo, J., Chrtek, J. Jr., Chytrý, M., Jarošík, V., Kaplan, Z., Krahulec, F., Moravcová, L., Pergl, J., Štajerová, K., Tichý, L. (2012a). Catalogue of alien plants of the Czech Republic (2nd edition): checklist update, taxonomic diversity and invasion patterns. Preslia 84: 155-255.

Pyšek, P., Chytrý, M., Pergl, J., Sádlo, J., Wild, J. (2012b). Plant invasions in the Czech Republic: current state, introduction dynamics, invasive species and invaded habitats. Preslia 84: 575-629.

Richardson, D., Holmes, P.M., Elser, K.J., Galatowitsch, S.M., Stromberg, J.C., Kirkman, S.P., Pyšek, P., Hobbs, R.J. (2007). Riparian vegetation: degradation, alien plant invasions, and restoration prospects. Diversity and Distributions, 13: 126-139.

Řepka, R., Maděra, P. (2009a). Rozšíření adventivních druhů v nížinných luzích jižní Moravy - případ hvězdnice kopinaté (Aster lanceolatus). In. Měkotová, J. (ed.): Řičñi krajina 6 (pp. 100-106). Sborník př́ispěvků z konference 21. ř́ijna 2009, Olomouc.

Řepka, R., Maděra, P. (2009b). Diverzita vyšších cévnatých rostlin lužního lesa ve vztahu k jeho věku. Zprávy České Botanické Společnosti, 44, Materiály 24: 101-110.

Řepka, R., Šebesta, J., Maděra, P., Koutecký, T., Klimánek, M. (2013). Druhová bohatost cévnatých rostlin lužních lesů dolního Podyjí. Mendel University, Brno, 124 p.

Řepka, R., Šebesta, J., Maděra, P., Vahalík, P. (2015). Comparison of the floodplain forest floristic composition of two riparian corridors: species richness, alien species and the effect of water regime changes. Biologia, 70 (2): 208-217.

Saccone, P., Brun, J.J., Michalet, R. (2010). Challenging growth-survival trade-off: A key for Acer negundo invasion in European floodplains? Canadian Journal of Forest Research, 40, 10: 1879-1886.

Santos, M.J. (2010). Encroachment of upland Mediterranean plant species in riparian ecosystems of southern Portugal. Biodiversity and Conservation, 19:2667-2684.

Schnitzler, A. (1994). Conservation of biodiversity in alluvial hardwood forests of the temperate zone. The example of Rhine valley. Forest ecology and management, 68: 385398.

Schnitzler, A. (1997). River dynamics as a forest process: interaction between fluvial systems and alluvial forests in large European river plains. The botanical review, 63, 1: 4064.

Schnitzler, A., Hale, B.W., Alsum, E.M. (2007). Examining native and exotic species diversity in European riparian forests. Biological Conservation, 138: 146-156.

Shafroth, P.B, Stromberg, J.C, Patten, D.T (2002). Riparian vegetation response to altered disturbance and stress regimes. Ecological Applications 12(1):107-123.

Stohlgren, T. J., Bull, K. A., Otsuki, Y., Villa, C. A., Lee, M. (1998): Riparian zones are havens for exotic plant species in the central grasslands. Plant Ecology, 138: 113-125.

Stokes, K., Ward, K., Colloff, M. (2010). Alterations in flood frequency increase exotic and native species richness of understorey vegetation in a temperate floodplain eucalypt forest. Plant Ecology, 211: 219-233.

Suchomel, J., Lusk, S., Macháček, P., Šebela, M. (2017). Červená kniha ohrožených druhů obratlovců lužnich lesů Biosférické rezervace Dolní Morava. Mendel University, Brno, 216 p. 
Šebela, M. (2004). Obojživelníci a plazi lužního lesa. In. In. Hrib M., Kordiovský E. (eds.): Lužní les v Dyjsko-moravské nivě (pp. 373-394). Moraviapress, Břeclav.

Tabacchi, E., Planty-Tabacchi, A.M., Salinas, M.J., Decamps, H. (1996). Landscape structure and diversity in riparian plant communities: a longitudinal comparative study. Regulated Rivers: Research \& Management, 12: 367-390.

Tremolieres, M., Sanchez-Perez, J.M., Schnitzler, A., Schmitt, D. (1998). Impact of river management history on the community structure, species composition and nutrient status in the Rhine alluvial hardwood forest. Plant Ecology 135: 59-78.

Trinajstič, I., Franjič, J., Škvorc, Ž. (2005). The flora of floodplain and marshy forests. In: Vukelič J. (ed.): Floodplain Forests in Croatia, (p. 93-101), Academy of Forestry Sciences, Zagreb.

Unar, P., Šamonil, P. (2008). The evolution of natural floodplain forests in South Moravia between 1973 and 2005. Journal of Forest Science, 54 (8): 340-354.

Úradníček, L., Maděra, P., Tichá, S., Koblížek, J. (2010). Woody Plants of the Czech Republic. Lesnická práce, Kostelec nad Černými lesy, 368 p.

Vašíček, F. (1985). Changes in the herbal vegetation along the topographical moisture gradient. In: Penka M., Vyskyt M, Klimo E., Vašíček F.: Floodplain Forests Ecosystem 2 (pp. 355-386). Academia, Praha.

Vicherek, J. et al. (2000). Flóra a vegetace na soutoku Moravy a Dyje. Masarykova univerzita v Brně, Brno, 368 p.

Viewegh, J. (2002). South-Moravian floodplain forest herb vegetation in the period 1978 1997. Journal of Forest Science, 48: 88-92.

Vrška, T. (1997). Prales Cahnov po 21 letech (1973-1994). Lesnictvi-Forestry 43: 155-180.

Vrška, T., (1998). Prales Ranšpurk po 21 letech (1973-1994). Lesnictví-Forestry, 44: 440473.

Vybíral, J., Hrib, M. (2000). Floodplain forests restoration at the Židlochovice Forest Enterprise. Lesy České republiky s.p. 10 p.

Ward, J.V., Tockner, K., Arscott, D.B., Claret, C. (2002). Riverine landscape diversity. Freshwater Biology, 47: 517-539.

Williams, CH.E. (2010). Survey of the alien flora of the Allegheny river island wilderness, Pennsylvania. Rhodora, 112, 950: 142-155.

Wenger, E.L., Zinke, A., Gutzweiler, K.A., (1990). Present situation of the European floodplain forests. Forest Ecology and Management, 33-34: 5-12. 